\title{
Lyapunov minimizing measures for expanding maps of the circle
}

\author{
G. CONTRERAS $\dagger$, A. O. LOPES $\ddagger$ and PH. THIEULLEN \\ $\dagger$ CIMAT, PO Box 402, 36000 Guanajuato, GTO, México \\ † Instituto de Matemática, UFRGS, Porto Alegre 91501-970, Brasil \\ § Département de Mathématiques, Université Paris-Sud, 91405 Orsay, France \\ (e-mail: thieu@topo.math.u-psud.fr)
}

(Received 17 March 1999 and accepted in revised form 17 June 2000)

\begin{abstract}
We consider the set of maps $f \in \mathcal{F}_{\alpha+}=\cup_{\beta>\alpha} \mathcal{C}^{1+\beta}$ of the circle which are covering maps of degree $D$, expanding, $\min _{x \in S^{1}} f^{\prime}(x)>1$ and orientation preserving. We are interested in characterizing the set of such maps $f$ which admit a unique $f$-invariant probability measure $\mu$ minimizing $\int \ln f^{\prime} d \mu$ over all $f$-invariant probability measures. We show there exists a set $\mathcal{G}_{+} \subset \mathcal{F}_{\alpha+}$, open and dense in the $\mathcal{C}^{1+\alpha}$-topology, admitting a unique minimizing measure supported on a periodic orbit. We also show that, if $f$ admits a minimizing measure not supported on a finite set of periodic points, then $f$ is a limit in the $\mathcal{C}^{1+\alpha}$-topology of maps admitting a unique minimizing measure supported on a strictly ergodic set of positive topological entropy.

We use in an essential way a sub-cohomological equation to produce the perturbation. In the context of Lagrangian systems, the analogous equation was introduced by R. Mañé and A. Fathi extended it to the all configuration space in [8].

We will also present some results on the set of $f$-invariant measures $\mu$ maximizing $\int A d \mu$ for a fixed $\mathcal{C}^{1}$-expanding map $f$ and a general potential $A$, not necessarily equal to $-\ln f^{\prime}$.
\end{abstract}

\section{Introduction}

We consider the space $\mathcal{F}_{\alpha}$ of $\mathcal{C}^{1+\alpha}$ maps of the circle $f: S^{1} \rightarrow S^{1}$ (where $\alpha<1$ ) which are covering maps of degree $D$, orientation-preserving and expanding:

$$
\lambda(f)=\min _{x \in S^{1}} f^{\prime}(x)>1 .
$$

We denote by $\operatorname{Höl}_{\alpha}(A)$ the $\alpha$-Hölder constant of a function $A: S^{1} \rightarrow \mathbb{R}$,

$$
\mathrm{Höl}_{\alpha}(A)=\sup _{0<d(x, y)<\pi}\left\{\frac{|A(x)-A(y)|}{d(x, y)^{\alpha}}\right\}
$$


by $\|A\|_{0}$ the uniform norm and by $\|A\|_{\alpha}$ the $\alpha$-Hölder norm of $A$,

$$
\|A\|_{\alpha}=\operatorname{Höl}_{\alpha}(A)+\|A\|_{0} .
$$

We note $\mathcal{C}^{\alpha}$, the set of $\alpha$-Hölder functions, and $\mathcal{C}^{\alpha+}$, the set $\cup_{\beta>\alpha} \mathcal{C}^{\beta}$ equipped with the $\mathcal{C}^{\alpha}$ topology. The set of $\mathcal{C}^{1+\alpha}$ maps of the circle is endowed with the distance

$$
d(f, g)=\|\bar{f}-\bar{g}\|_{0}+\left\|f^{\prime}-g^{\prime}\right\|_{\alpha}
$$

where $\bar{f}, \bar{g}$ denote the covering maps of $f, g$ to $\mathbb{R}$ fixing zero. The space $\mathcal{C}^{1+\alpha}$ becomes a complete space and $\mathcal{F}_{\alpha}$ an open set of $\mathcal{C}^{1+\alpha}$. We denote by $\mathcal{F}_{\alpha+}$ the set $\cup_{\beta>\alpha} \mathcal{F}_{\beta}$ equipped with the $\mathcal{C}^{1+\alpha}$-topology. Although $\mathcal{C}^{2}$ maps are dense in $\mathcal{C}^{1}$ for the $\mathcal{C}^{1}$-topology, the closure of $\mathcal{C}^{\alpha+}$ for the $\mathcal{C}^{\alpha}$-topology is strictly included in $\mathcal{C}^{\alpha}$.

Definition 1. Let $\mathcal{K}(f)$ be the set of $f$-invariant probability measures,

$$
\begin{gathered}
J(f)=\inf \left\{\exp \left(\int \ln f^{\prime} d \nu\right) \mid v \in \mathcal{K}(f)\right\} \\
\mathcal{M}(f)=\left\{\mu \in \mathcal{K}(f) \mid \int \ln f^{\prime} d \mu=\ln J(f)\right\} .
\end{gathered}
$$

A measure in $\mathcal{M}(f)$ is called a Lyapunov minimizing measure.

The purpose of this paper is to show the following theorem. We recall first that a compact invariant set is said to be strictly ergodic if it is minimal and uniquely ergodic or, equivalently, if it is uniquely ergodic and the support of the unique invariant measure is equal to the compact set itself.

THEOREM 2. Let $\alpha<1$.

(i) The set $\mathcal{G}_{+}$of maps $f$ in $\mathcal{F}_{\alpha+}$ having a unique Lyapunov minimizing measure supported on a periodic orbit and satisfying the property of continuously varying support is open and dense in $\mathcal{F}_{\alpha+}$.

(ii) If $f \in \mathcal{F}_{\alpha+}$ has a Lyapunov minimizing measure not supported on a finite set of periodic points, then $f$ is a limit in the $\mathcal{C}^{1+\alpha}$-topology of maps $\left(f_{n}\right)$ in $\mathcal{F}_{\alpha+}$ admitting a unique Lyapunov minimizing measure $\mu_{n}$ such that $f_{n}$ restricted to $\operatorname{supp}\left(\mu_{n}\right)$ is strictly ergodic and has positive topological entropy.

The property of continuously varying support was first introduced by Mañé in the Lagrangian setting. It can be formulated in our setting in the following way.

Definition 3. Suppose $f \in \mathcal{F}_{\alpha}$ admits a unique Lyapunov minimizing measure $\mu$. We say that $f$ satisfies the property of continuously varying support if, for any sequence $\left(f_{n}\right)$ of $\mathcal{F}_{\alpha}$ converging to $f$ in the $\mathcal{C}^{1+\alpha}$-topology, for any Lyapunov minimizing measures $\mu_{n}$ for $f_{n}$, the sequence $\left(\mu_{n}\right)$ converges to $\mu$ in the weak topology and the sequence of compact sets $\left(\operatorname{supp}\left(\mu_{n}\right)\right)$ converges to $\operatorname{supp}(\mu)$ in the Hausdorff topology.

The problem we consider here is in some sense analogous (although we do not consider the homological position) to the problems considered in Aubry-Mather theory $($ see $[\mathbf{6}, \mathbf{1 8}]$ ) for Lagrangian flows. A recent result of Mañé (see $[\mathbf{1 8}]$ and also $[\mathbf{5 , 6 ]}$ ) on Lagrangian flows shows that generically on the Lagrangian there is a unique measure minimizing 
action. The main difference of these results to our setting is that the minimization in our theorem is among invariant probabilities and in Mañé's theorem the minimization can be done among a fixed subset of probability measures (not necessarily invariant) independent of $L$. Therefore, in the setting considered here, if we change the map, the set of invariant probabilities will also change and this is one of the main differences from [18] to our proof. The above theorem is the analogous result for the setting of expanding maps of a conjecture for Lagrangians proposed by Mañé in $[\mathbf{1 8}, \mathbf{1 9}]$. Ott also proposed a related conjecture (see [12]). In the Lagrangian case, the conjecture whether, generically on the Lagrangian, the unique maximizing measure is supported on a periodic orbit is still open. Lyapunov minimizing measures have been previously considered by [12-15, 22].

In our setting, the set $\mathcal{M}(f)$ may not be reduced to a single probability measure and the more pathological case is when $\ln f^{\prime}$ is cohomologous to a constant $\ln D$, that is, when there exists a positive function $h: S^{1} \rightarrow \mathbb{R}$ such that $f^{\prime}=D(h \circ f / h)$. In that case, $J(f)=D$ and any invariant measure is minimizing. See Proposition 28 for equivalent properties for $\ln f^{\prime}$ to be cohomologous to a constant. Nevertheless, $\ln f^{\prime}$ always satisfies a sub-cohomological equation which is one of the key ingredients of the proof of Theorem 2 .

THEOREM 4. For any $f \in \mathcal{F}_{\alpha}$, there exists a $\alpha$-Hölder function $\left.\left.h: S^{1} \rightarrow\right] 0,1\right]$ such that $f^{\prime} \geq J(f)(h \circ f / h)$ everywhere on $S^{1}$ and $f^{\prime}=J(f)(h \circ f / h)$ on the support of any Lyapunov minimizing measures.

The proof of Theorem 2 depends actually on similar statements where $f$ is fixed and $A=-\log f^{\prime}$ varies among all Hölder functions with zero pressure. We therefore develop in $\$ 2$ a theory for maximizing a general potential $A$ (not necessarily of zero pressure).

Definition 5. For any $\mathcal{C}^{1}$-expanding map $f$ and any Hölder function defined on $S^{1}, A$ : $S^{1} \rightarrow \mathbb{R}$, we call

$$
\begin{aligned}
& m(A, f)=\sup \left\{\int A d v \mid v \in \mathcal{K}(f)\right\} \\
& \mathcal{M}(A, f)=\left\{\mu \in \mathcal{K}(f) \mid \int A d \mu=m(A, f)\right\} .
\end{aligned}
$$

Any measure in $\mathcal{M}(A, f)$ will be called a maximizing measure for $(A, f)$.

As usual, a set is said to be generic if it contains a countable intersection of open and dense sets.

THEOREM 6. Let $f$ be a $\mathcal{C}^{1}$-expanding map and $\alpha>0$. Then the set of $\alpha$-Hölder functions $A$ admitting a unique maximizing measure for $(A, f)$ is generic in $\mathcal{C}^{\alpha}$. For such functions $A$, the map $f$ is strictly ergodic on the support of its unique maximizing measure.

If $A$ is $\mathcal{C}^{0}$ and has a unique maximizing measure $\mu$, if $\left(A_{n}\right)$ converges to $A$ in the $\mathcal{C}^{0}$ topology and $\mu_{n}$ is chosen in $\mathcal{M}\left(A_{n}, f\right)$ for all $n$, then $\left(\mu_{n}\right)$ converges to $\mu$ in the weak topology (see step one in the proof of Proposition 16). In the following definition we ask a stronger property.

Definition 7. Let $f$ be a $\mathcal{C}^{1}$-expanding map and let $A$ be a $\alpha$-Hölder function admitting a unique maximizing measure $\mu$. We say $A$ satisfies the property of continuously varying 
support if, for any sequence $\left(A_{n}\right)$ of $\alpha$-Hölder functions converging to $A$ in the $\alpha$-Hölder topology and for any measure $\mu_{n}$ chosen in $\mathcal{M}\left(A_{n}, f\right)$, the sequence of measures $\left(\mu_{n}\right)$ converges weakly to $\mu$ and the sequence of its compact supports $\left(\operatorname{supp} \mu_{n}\right)$ converges to supp $\mu$ in the Hausdorff topology.

In the case when the unique maximizing measure $\mu$ is supported by a single orbit, because the map $f$ is expanding, the property of continuously varying support is equivalent to saying there exists a neighborhood $\mathcal{U}$ of $A$ in the $\mathcal{C}^{\alpha}$-topology such that, for all $B$ in $\mathcal{U}$, $\mathcal{M}(B, f)=\mathcal{M}(A, f)=\{\mu\}$.

THEOREM 8. Let $f$ be a $\mathcal{C}^{1}$-expanding map.

(i) The set of $A \in \mathcal{C}^{\alpha}$ having a unique maximizing measure $\mu_{A}$ satisfying the property of continuously varying support is generic in $\mathcal{C}^{\alpha}$.

(ii) The set $\mathcal{G}$ of $A \in \mathcal{C}^{\alpha}$ having a unique maximizing measure supported on a periodic orbit and satisfying the property of continuously varying support is open in $\mathcal{C}^{\alpha}$ and $\mathcal{G}_{+}=\mathcal{G} \cap \mathcal{C}^{\alpha+}$ is open and dense in $\mathcal{C}^{\alpha+}$.

(iii) Let $A \in \mathcal{C}^{\alpha+}$ be fixed. If $\mathcal{M}(A, f)$ contains a maximizing measure which is not supported on a finite set of periodic points then there exists a sequence $\left(B_{n}\right)$ converging to $A$ in the $\mathcal{C}^{\alpha}$-topology such that each $B_{n}$ has a unique maximizing measure supported on a strictly ergodic invariant compact set of positive entropy and satisfying the property of continuously varying support.

As before, the main tool to prove Theorem 8 is the following sub-cohomological equation. For a generalization to smooth Anosov diffeomorphisms, see [23].

THEOREM 9. Let $f$ be a $\mathcal{C}^{1}$-expanding map and $A \in \mathcal{C}^{\alpha}$. Then there exists $V \in \mathcal{C}^{\alpha}$ such that $A \leq V \circ f-V+m(A, f)$. In particular, $A$ is cohomologous to $m(A, f)$ on the support of any maximizing measure $\mu \in \mathcal{M}(A, f)$, that is, $A=V \circ f-V+m(A, f)$ on the support of $\mu$.

The function $V$ should be called sub-action in analogy with Lagrangian mechanics, KAM theory and with $[\mathbf{8}]$, because it corresponds, for the discrete time version, to a subsolution of the Hamilton-Jacobi equation:

$$
S(\gamma(1))-S(\gamma(0)) \leq \int_{0}^{1} L d t-E,
$$

where $L$ is the Lagrangian restricted to $\gamma(t), t \in(0,1)$, and $E$ is the energy. The function $A$ plays the role of the Lagrangian, $m(A, f)$ plays the role of energy, $f$ plays the role of the flow and the inequality is inverted because we are maximizing and not minimizing.

The strategy of the proof of the above results is the following. In $\$ 2$ we prove Theorems 6, 8 and 9 for a fixed expanding maps $f \in \mathcal{F}_{0}$. We then transfer in $\S 3$ the above properties to prove Theorems 2 and 4 for varying functions $f$. In that case, the potential $A$ is linked to the function by the formula $A=-\log f^{\prime}$ and the thermodynamic formalism is used. Analogous results hold for topologically mixing one-sided sub-shifts of finite type in $D$-symbols and by means of a Markov partition to general mixing expanding maps on a compact set (see [4] for the construction of such a Markov partition). 


\section{Maximizing measures for Hölder potentials}

We begin by proving that, generically, there exists a unique maximizing measure. This comes mainly from the fact that, for a compact convex set in $\mathbb{R}^{N}$, among the set of hyperplanes which support the convex set, the set of those hyperplanes having an intersection reduced to a single point is generic. Nonetheless, the proof has to be carried in infinite dimension and requires more details.

We first recall some definitions. We say that a point $p$ is an extremal point of a compact convex set $C$ of $\mathbb{R}^{n}$ if $p$ is not the mid point of a segment totally included in $C$. We say that $p$ is strictly extremal if there exists a linear form which attains its maximum at the point $p$ only. A classical result (see [21]) states that $C$ is equal to the closed convex hull of its strictly extremal points. Theorem 6 is a direct consequence of the one where $\mathcal{K}=\mathcal{K}(f)$ and $\mathcal{H}=\mathcal{C}^{\alpha}$.

PROPOSITION 10. Let $\mathcal{K}$ be a compact convex subset of the set of probability measures on $S^{1}$ and $\left(\mathcal{H},\|\cdot\|_{\mathcal{H}}\right)$ be a dense Banach space in $\mathcal{C}^{0}\left(S^{1}, \mathbb{R}\right)$ which embeds continuously in $\mathcal{C}^{0}\left(S^{1}, \mathbb{R}\right)$. Then there exists a residual set $\mathcal{R}$ in $\mathcal{H}$ (for the $\|\cdot\|_{\mathcal{H}}$-topology) such that, for all $A \in \mathcal{R}$, if

$$
\mathcal{M}(A) \stackrel{\text { def }}{=}\left\{\mu \in \mathcal{K} \mid \int A d \mu=m(A)\right\} \quad \text { and } m(A) \stackrel{\text { def }}{=} \max \left\{\int A d \mu \mid \mu \in \mathcal{K}\right\}
$$

then $\mathcal{M}(A)$ contains a unique measure.

Proof. Let $\left\{H_{n}\right\}_{n>1}$ be a dense subset of the unit ball of $\mathcal{H}$. Since $\mathcal{H}$ is dense

$$
d\left(\mu, \mu^{\prime}\right) \stackrel{\text { def }}{=} \sum_{n \geq 1} \frac{1}{2^{n}}\left|\int H_{n} d \mu-\int H_{n} d \mu^{\prime}\right|
$$

defines a metric on $\mathcal{K}$ compatible with the weak topology. Let us call

$$
\mathcal{R}_{\epsilon} \stackrel{\text { def }}{=}\left\{A \in \mathcal{C}^{0}\left(S^{1}, \mathbb{R}\right) \mid \operatorname{diam} \mathcal{M}(A)<\epsilon\right\} .
$$

We claim that $\mathcal{R}_{\epsilon}$ is open in $\mathcal{C}^{0}\left(S^{1}, \mathbb{R}\right)$ and $\mathcal{R}_{\epsilon} \cap \mathcal{H}$ is dense in $\mathcal{H}$ for the $\|\cdot\|_{\mathcal{H}}$-topology. The desired residual set will be $\mathcal{R}=\cap_{\epsilon} \mathcal{R}_{\epsilon} \cap \mathcal{H}$.

We show by contradiction that $\mathcal{R}_{\epsilon}$ is open. If not, one can find $A$ in $\mathcal{R}_{\epsilon}, B_{n}$ in $\mathcal{C}^{0}\left(S^{1}, \mathbb{R}\right)$ and $\left(\mu_{n}, v_{n}\right)$ in $\mathcal{M}\left(A+B_{n}\right)$ such that $\left\|B_{n}\right\|_{0}$ converges to zero and $d\left(\mu_{n}, v_{n}\right) \geq \epsilon$ for all $n$. We may assume by taking a subsequence that $\left(\mu_{n}\right)$ and $\left(v_{n}\right)$ converge to $\bar{\mu}$ and $\bar{\nu}$. Let us prove that $\bar{\mu} \in \mathcal{M}(A)$ : indeed for every $\mu \in \mathcal{K}$,

$$
\int\left(A+B_{n}\right) d \mu \leq \int\left(A+B_{n}\right) d \mu_{n} \leq \int A d \mu_{n}+\left\|B_{n}\right\|_{0}
$$

and $\int A d \mu \leq \int A d \bar{\mu}$ by taking the limit on $n$. For the same reason $\bar{v}$ belongs to $\mathcal{M}(A)$. We have obtained a contradiction since $d(\bar{\mu}, \bar{v}) \geq \epsilon$.

We now show that $\mathcal{R}_{\epsilon} \cap \mathcal{H}$ is dense in $\mathcal{H}$. Let $A_{0} \in \mathcal{H}$ and $\mathcal{K}_{0}=\mathcal{M}\left(A_{0}\right)$. The continuous projections $\pi_{n}: \mathcal{K} \rightarrow \mathbb{R}^{n}, \pi_{n}(\mu)=\left(\int H_{1} d \mu, \ldots, \int H_{n} d \mu\right)$ sends $\mathcal{K}_{0}$ to a compact convex set $\pi_{n}\left(\mathcal{K}_{0}\right)$ which admits a strictly extremal point $p_{n}$. We first notice that diam $\pi_{n}^{-1}\left(p_{n}\right) \leq 2^{-n}$ and we choose $n$ large enough so that $2^{-n}<\epsilon$. By definition of $p_{n}=\left(p^{1}, \ldots, p^{n}\right)$ there exists $\left(a^{1}, \ldots, a^{n}\right) \in \mathbb{R}^{n}$ such that

$$
\sum_{i=1}^{n} a^{i} p^{i}>\sum_{i=1}^{n} a^{i} q^{i} \quad \forall q=\left(q^{1}, \ldots, q^{n}\right) \in \pi_{n}\left(K_{0}\right), q \neq p .
$$


In particular, if $A_{1}=\sum_{i=1}^{n} a^{i} H_{i}, m_{0}\left(A_{1}\right)=\max \left\{\int A_{1} d \mu \mid \mu \in \mathcal{K}_{0}\right\}$ and

$$
\mathcal{M}_{0}\left(A_{1}\right) \stackrel{\text { def }}{=}\left\{\mu \in K_{0} \mid \int A_{1} d \mu=m_{0}\left(A_{1}\right)\right\}
$$

then $\mathcal{M}_{0}\left(A_{1}\right)=\pi_{n}^{-1}\left(p_{n}\right)$ has diameter less than $\epsilon$. We show that, for small enough $\zeta>0, A_{\zeta}=(1-\zeta) A_{0}+\zeta A_{1} \in \mathcal{R}_{\epsilon}$. More precisely we show that, for any open set $\mathcal{U} \supset \mathcal{M}_{0}\left(A_{1}\right)$, for any $\zeta$ sufficiently small, $\mathcal{M}\left(A_{\zeta}\right) \subset \mathcal{U}$. By contradiction, there exists a sequence $\mu_{n} \in \mathcal{M}\left(A_{\zeta_{n}}\right) \backslash \mathcal{U}$ for some $\zeta_{n}$ converging to zero. We may assume that $\left(\mu_{n}\right)$ converges to $\bar{\mu} \in \mathcal{K} \backslash \mathcal{U}$. We first show that $\bar{\mu} \in \mathcal{K}_{0}=\mathcal{M}\left(A_{0}\right)$ : for every $\mu \in \mathcal{K}$,

$$
\int A_{\zeta_{n}} d \mu \leq \int A_{\zeta_{n}} d \mu_{n} \leq \int A_{0} d \mu_{n}+\zeta_{n}\left\|A_{1}-A_{0}\right\|_{0}
$$

and by taking a limit in $n, \int A_{0} d \mu \leq \int A_{0} d \bar{\mu}$. We then show that $\bar{\mu}$ belongs to $\mathcal{M}_{0}\left(A_{1}\right)$ : for every $\mu \in \mathcal{K}_{0}$,

$$
\begin{aligned}
\int A_{\zeta_{n}} d \mu & =\left(1-\zeta_{n}\right) \int A_{0} d \mu+\zeta_{n} \int A_{1} d \mu \\
& \leq\left(1-\zeta_{n}\right) \int A_{0} d \mu_{n}+\zeta_{n} \int A_{1} d \mu_{n}
\end{aligned}
$$

Since $\int A_{0} d \mu_{n} \leq \int A_{0} d \mu$, we have obtained $\int A_{1} d \mu \leq \int A_{1} d \mu_{n}$ and at the limit $\int A_{1} d \mu \leq \int A_{1} d \bar{\mu}$. We have obtained a contradiction since $\bar{\mu} \notin \mathcal{U}$.

We now prove the cocycle Theorem 9. We show that any $A \in \mathcal{C}^{\alpha}$ is sub-cohomologous to a constant $m(A, f)$ with a unique minimal coboundary $V_{A}$. The main tool is the shadowing lemma. We say that two points $x$ and $y$ belong to the same inverse branch of length $n$ if there exists a close interval $I$ containing $x$ and $y$ such that $f^{n}$ restricted to $\operatorname{int}(I)$ is one-to-one and $f^{n}(I)=S^{1}$. Theorem 9 is strongly related to Theorem B of Mañé [17]. Our result is in some sense stronger since we obtain a sub-coboundary defined everywhere (this fact is crucial in the following) whereas Mañé's proof gives a coboundary defined only on the support of the maximizing measure.

Proposition 11. Let $f$ be a $\mathcal{C}^{1}$-expanding map and $A \in \mathcal{C}^{\alpha}$. Then there exists a unique minimal non-negative $\alpha$-Hölder function $V_{A}: S^{1} \rightarrow \mathbb{R}$ such that:

(i) $A \leq V_{A} \circ f-V_{A}+m(A, f)$ on $S^{1}$;

(ii) for any non-negative function $W: S^{1} \rightarrow \mathbb{R}$ (not necessarily Hölder) satisfying $A \leq W \circ f-W+m(A, f)$ on $S^{1}$, we have $V_{A} \leq W$ on $S^{1}$.

Moreover, $\operatorname{Höl}_{\alpha}\left(V_{A}\right) \leq \operatorname{Höl}_{\alpha}(A) /\left(\lambda^{\alpha}-1\right)$ and $A=V_{A} \circ f-V_{A}+m(A, f)$ everywhere on $\operatorname{supp}(\mu)$ of any maximizing measure $\mu$ for $(A, f)$.

Proof. We may assume $m(A, f)=0$. We recall that $S_{n} A$ denotes the Birkhoff sum $\sum_{k=0}^{n-1} A \circ f^{k}$ and that $S_{n} A=0$ when $n=0$. Let us define

$$
V_{A}(x)=\sup \left\{S_{n} A(y) \mid n \geq 0 \text { and } f^{n}(y)=x\right\} .
$$

We show that $V_{A}$ is well defined. On the one hand, for any periodic point $p$ of period $n$, $S_{n} A(p) \leq 0$. On the other hand, for any $n \geq 0$ and any $y \in S^{1}$ there exists a periodic 
point $p$ in the same inverse branch of length $n$ as $y$ such that $f^{n}(p)=p$. Moreover, if $N$ is large enough, $\lambda^{N}>2$, for all $k<n-N, d\left(f^{k}(y), f^{k}(p)\right)<\frac{1}{2}$ and

$$
\left|S_{n} A(y)-S_{n} A(p)\right| \leq \frac{\operatorname{Höl}_{\alpha}(A)}{\lambda^{\alpha}-1}+2 N\|A\|_{0} .
$$

We now show that $V_{A}$ is $\alpha$-Hölder. If $f^{n}(y)=x, f^{n}\left(y^{\prime}\right)=x^{\prime}$ and $y$ and $y^{\prime}$ are in the same inverse branch of length $n$, if $d\left(x, x^{\prime}\right)<\frac{1}{2}$ then

$$
\left|S_{n} A(y)-S_{n} A\left(y^{\prime}\right)\right| \leq \frac{\operatorname{Höl}_{\alpha}(A)}{\lambda^{\alpha}-1} d\left(x, x^{\prime}\right)^{\alpha}
$$

and, by taking the supremum over $y$ and $n$, we finally obtain

$$
\left|V_{A}(x)-V_{A}\left(x^{\prime}\right)\right| \leq \frac{\operatorname{Höl}_{\alpha}(A)}{\lambda^{\alpha}-1} d\left(x, x^{\prime}\right)^{\alpha} .
$$

By definition of $V_{A}$, we have $V_{A} \circ f \geq A+V_{A}$ on $S^{1}$. If $W$ is non-negative and satisfies $W \circ f \geq A+W$ on $S^{1}$, then for every $n \geq 0,(x, y)$ such that $f^{n}(y)=x$, $W(x) \geq S_{n} A(y)+W(y)$ and therefore $W(x) \geq V_{A}(x)$. If $\mu$ is a maximizing measure, $\int\left(V_{A} \circ f-V_{A}-A\right) d \mu=0$ and $V_{A} \circ f-V_{A}=A$ on $\operatorname{supp}(\mu)$.

Remark 12. We collect here alternative proofs of the existence of a sub-coboundary.

(i) If we choose in Proposition 11 the following definition of $\tilde{V}_{A}$ :

$$
\tilde{V}_{A}(x)=\lim _{n \rightarrow \infty} \sup _{k>n} \sup _{f^{k}(y)=x}\left\{S_{k} A(y)-k m(A, f)\right\},
$$

we again obtain a sub-coboundary which satisfies in addition the functional equation considered by Bousch [2] and Fathi [8]:

$$
\forall x \in S^{1}, \quad \tilde{V}_{A}(x)=\max _{f(y)=x}\left\{A(y)-m(A, f)+\tilde{V}_{A}(y)\right\} .
$$

In particular $(D=2)$, for any opposite points $x, x^{\prime}$ (i.e. $\left.f(x)=f\left(x^{\prime}\right)\right)$, one of them belongs to the set $\Sigma=\left\{A-m(A, f)=\tilde{V}_{A} \circ f-\tilde{V}_{A}\right\}$. $\Sigma$ has therefore always a non-empty interior and $x \in \partial \Sigma \Rightarrow x^{\prime} \in \Sigma$.

A second way to prove the existence of a sub-coboundary is to use Proposition 23(iii) where we define an action potential $S_{A}(x, y)$. A third way is to use the thermodynamic formalism: see Proposition 29(iii).

(ii) If $A$ is not equal to a coboundary modulo a constant then the set of $\alpha$-Hölder functions $W$ which are solutions of $A \leq W \circ f-W+m(A, f)$ is always a large set. Indeed let $R=V_{A} \circ f-V_{A}-A+m(A, f)$ and $1 \geq \alpha_{0} \geq \alpha_{1} \cdots \geq \alpha_{n} \geq 0$, $\alpha$-Hölder functions, then

$$
W=V_{A}+\left(\alpha_{0} R\right)+\left(\alpha_{1} R\right) \circ f+\cdots+\left(\alpha_{n} R\right) \circ f^{n}
$$

is such a solution.

(iii) Proposition 11 shows that, in the case when $A$ admits a unique maximizing measure $\mu, f$ is uniquely ergodic on $\operatorname{supp}(\mu)$. This follows from the fact that any measure $v$ with support contained in the support of $\mu$ satisfies $\int A d v=m(A, f)$. A maximizing measure for $A$ may not be unique: for instance, when $A$ is of the form $A=m+V \circ f-V$, any invariant measure is maximizing. 
In order to prove the property of continuously varying support, we introduce a vocabulary close to what is used in the setting of Lagrangian flows.

Definition 13. Let $A$ be a continuous function and $m=m(A, f)$.

(i) A function $W$ is called a sub-action for $(A, f)$ if it satisfies the inequality $A-m \leq$ $W \circ f-W$ everywhere. We then call the set

$$
\Sigma_{A, W}=\left\{x \in S^{1} \mid A(x)-m=W \circ f(x)-W(x)\right\}
$$

the $W$-action set (we could have called it the Mañé set).

(ii) If $W$ is a sub-action and $(x, y)$ is a pair of points of $S^{1}$, we say that the points $(x, y)$ are $W$-connected and we write $x \stackrel{W}{\rightarrow} y$ if, for every $\epsilon>0$, there exist $z \in S^{1}$ and $n \geq 1$ such that $d(z, x)<\epsilon, f^{n}(z)=y$ and

$$
\left|S_{n}(A-m)(z)-(W(y)-W(x))\right|<\epsilon .
$$

We say that $(x, y)$ are $W$-equivalent if $x \stackrel{W}{\rightarrow} y$ and $y \stackrel{W}{\rightarrow} x$.

(iii) A point $x \in S^{1}$ is said to be non-wandering with respect to $(A, f)$ if, for any $\epsilon>0$, there exist $z \in S^{1}$ and $n \geq 1$ such that

$$
d(z, x)<\epsilon, f^{n}(z)=x \quad \text { and } \quad\left|S_{n}(A-m)(z)\right|<\epsilon .
$$

We denote by $\Omega(A, f)$ the set of non-wandering points with respect to $(A, f)$. We will see shortly that $\Omega(A, f)$ is not empty.

(iv) If $W$ is a sub-action, a compact invariant set $K$ (i.e. $f(K)=K$ ) is said to be $W$-irreducible if any two points $(x, y)$ of $K$ are $W$-equivalent.

We first give elementary properties. The main tool is the standard shadowing lemma. We recall that, for a complete orbit $\underline{x}=\left(x_{n}\right)_{n \in \mathbb{Z}}$ in $S^{1}\left(f\left(x_{n}\right)=x_{n+1}\right.$ for all $\left.n \in \mathbb{Z}\right), \omega(\underline{x})$ denotes the compact invariant set of accumulation points of $\left(x_{n}\right)_{n \geq 0}$ and $\alpha(\underline{x})$ the compact invariant set of accumulation points of $\left(x_{-n}\right)_{n \geq 0}$. We also recall that an $\epsilon$-pseudo orbit with $M$ jumps from $x$ to $y$ is a finite sequence of points $\left\{x_{0} \ldots x_{n}\right\}$ such that $x_{0}=x, x_{n}=y$, $d\left(f\left(x_{k}\right), x_{k+1}\right)<\epsilon$ for all $0 \leq k<n$, and the cardinality of the set of indices $0 \leq k<n$ satisfying $f\left(x_{k}\right) \neq x_{k+1}$ is bounded by $M$.

LEMMA 14. Let $W$ be a Hölder sub-action.

(i) Let $(x, y)$ be a pair of points and $M>0$ an integer. Then $x \stackrel{W}{\rightarrow} y$ if and only if, for every $\epsilon>0$, there exists a $\epsilon$-pseudo orbit with at most $M$ jumps from $x$ to $y$ such that

$$
\left|\sum_{k=0}^{n-1}(A-m(A, f))\left(x_{k}\right)-(W(y)-W(x))\right|<\epsilon .
$$

(ii) If $x \stackrel{W}{\rightarrow}$ y and $y \stackrel{W}{\rightarrow} z$ then $x \stackrel{W}{\rightarrow} z$.

(iii) Any $W$-irreducible compact invariant set $K$ is included in $\Omega(A, f)$.

(iv) If $\underline{x}=\left(x_{n}\right)_{n \in \mathbb{Z}}$ is a complete orbit in the $W$-action set, then $\alpha(\underline{x})$ and $\omega(\underline{x})$ are compact invariant $W$-irreducible sets and therefore belong to $\Omega(A, f)$. For any $\alpha \in \alpha(\underline{x})$ and $\omega \in \omega(\underline{x}), \alpha \stackrel{W}{\rightarrow} x \stackrel{W}{\rightarrow} \omega$. 
The main ingredients for proving Theorem 8 are given by the following two propositions. Proposition 15(ii) shows in particular that $\mathcal{M}(A, f)$ is equal to the set of all invariant measures whose support stays in $\Omega(A, f)$.

Proposition 15. Let A be a Hölder function. Then:

(i) $\quad \Omega(A, f)$ is a compact invariant set included in the $W$-action set of any continuous sub-action $W$.

(ii) For any invariant measure $\mu, \mu \in \mathcal{M}(A, f)$ if and only if $\operatorname{supp}(\mu) \subset \Omega(A, f)$. In particular, $(A, f)$ admits a unique maximizing measure, if and only if $\Omega(A, f)$ is uniquely ergodic. If $\mu$ is an ergodic maximizing measure, $\operatorname{supp}(\mu)$ is $W$-irreducible for any continuous sub-action $W$.

(iii) If $\Omega(A, f)$ is $W$-irreducible for some Hölder sub-action $W$, then $\Omega(A, f)$ contains all compact invariant sets included in the $W$-action set.

(iv) If $(A, f)$ admits a unique maximizing measure, then $\Omega(A, f)$ is $W$-irreducible for any Hölder sub-action $W$.

Proof. Part (i). If $\left(x_{i}\right)_{i \geq 0}$ is a sequence of points of $\Omega(A, f)$ converging to $x$ and $\epsilon>0$, then $d\left(x_{i}, x\right)<\frac{1}{2} \epsilon$ for some $i$ and there exist $y_{i}, n>0$ such that $d\left(y_{i}, x_{i}\right)<$ $\frac{1}{2} \epsilon, d\left(f^{n}\left(y_{i}\right), x_{i}\right)<\frac{1}{2} \epsilon$ and $\left|S_{n}(A-m(A, f))\left(y_{i}\right)\right|<\epsilon$. Then $d\left(y_{i}, x\right)<\epsilon$ and $d\left(f^{n}\left(y_{i}\right), x\right)<\epsilon$. We have proved $\Omega(A, f)$ is closed.

Let $x$ be a point of $\Omega(A, f)$ and $\epsilon>0$. We choose, using the continuity of $f$ and $A$, $\eta>0$ so that $f(B(x, \eta)) \subset B(f(x), \epsilon)$ and the oscillation of $A$ on $B(x, \eta)$ is bounded by $\epsilon$. Then there exist $y$ and $n>0$ such that $d(y, x)<\eta, d\left(f^{n}(y), x\right)<\eta$ and $\left|S_{n}(A-m(A, f))(y)\right|<\epsilon$. Using the definition of $\eta$, we obtain $d(f(y), f(x))<\epsilon$, $d\left(f^{n+1}(y), f(x)\right)<\epsilon$ and

$$
\left|S_{n}(A-m(A, f))(f(y))\right|=\left|S_{n}(A-m(A, f))(y)-\left(A-A \circ f^{n}\right)(y)\right|<2 \epsilon .
$$

We have proved $f(\Omega(A, f)) \subset \Omega(A, f)$.

If $x^{\prime} \in \Omega(A, f),\left(y_{i}^{\prime}\right)_{i \geq 0}$ is a sequence of points in $S^{1}$ converging to $x^{\prime},\left(n_{i}\right)_{i \geq 0}$ is an increasing sequence of integers such that $f^{n_{i}}\left(y_{i}^{\prime}\right)$ converges to $x^{\prime}$ and $\mid S_{n_{i}}(A-$ $m(A, f))\left(y_{i}^{\prime}\right) \mid$ converges to zero, by taking a subsequence we may assume $f^{n_{i}-1}\left(y_{i}^{\prime}\right)$ converges to some $x$ satisfying $f(x)=x^{\prime}$. For any large $i, y_{i}^{\prime}$ admits a unique pre-image $y_{i}$ close to $x$ and as above $\left|S_{n_{i}}(A-m(A, f))\left(y_{i}\right)\right|$ converges to zero. We have proved $f(\Omega(A, f))=\Omega(A, f)$.

To prove that $\Omega(A, f)$ is included in the $W$-action set, we introduce the non-negative function

$$
R_{A, W}=W \circ f-W+m(A, f)-A .
$$

If $x \in \Omega(A, f),\left(y_{i}\right)_{i \geq 0}$ converges to $x$ and $\left(n_{i}\right)_{i \geq 0}$ is chosen so that $f^{n_{i}}\left(y_{i}\right)$ converges to $x$ and $S_{n_{i}}(A-m(A, f))\left(y_{i}\right)$ converges to zero, then

$$
S_{n_{i}}(A-m(A, f))\left(y_{i}\right)+S_{n_{i}} R_{A, W}\left(y_{i}\right)=W \circ f^{n_{i}}\left(y_{i}\right)-W\left(y_{i}\right)
$$

converges to zero and therefore $R(x)=0$.

Part (ii). The fact that the support of any maximizing measure $\mu$ is included in $\Omega(A, f)$ follows from Atkinson's theorem [1] since, by definition of $\mu, \int(A-m(A, f)) d \mu=0$. 
If, in addition $\mu$ is ergodic, for any two points $(x, y)$ in $\operatorname{supp}(\mu)$, there exists a path $\left\{z, \ldots, f^{n}(z)\right\}$ included in the support of $\mu$ which connects as close as we want $(x, y)$. Since that path is included in the $W$-action set, we have just shown that $\operatorname{supp}(\mu)$ is $W$-irreducible.

Part (iii). We now assume that $\Omega(A, f)$ is $W$-irreducible for some Hölder sub-action $W$. Let $K$ be a compact invariant set included in the $W$-action set. We pick $\underline{z}=\left(z_{n}\right)_{n \in \mathbb{Z}}$, a complete orbit in $K$ passing through $z$ (i.e. $\left.z_{0}=z\right), \alpha \in \alpha(\underline{z})$ and $\omega \in \omega(\underline{z})$. Then $\alpha \stackrel{W}{\rightarrow} z \stackrel{W}{\rightarrow} \omega$ and by irreducibility of $\Omega(A, f), \omega \stackrel{W}{\rightarrow} \alpha$. Therefore, $z \stackrel{W}{\rightarrow} z$ and $z \in \Omega(A, f)$.

Part (iv). The proof is very similar to part (iii). Let $\mu$ be the unique maximizing measure, $x, y$, points of $\Omega(A, f)$, and $\underline{x}=\left(x_{n}\right)_{n \in \mathbb{Z}}, y=\left(y_{n}\right)_{n \in \mathbb{Z}}$ complete orbits passing through $x, y$, respectively. We define two sequences of probability measures:

$$
\omega_{n}=\frac{1}{n} \sum_{k=0}^{n-1} \delta_{x_{k}} \quad \text { and } \quad \alpha_{n}=\frac{1}{n} \sum_{k=0}^{n-1} \delta_{y_{-k}} .
$$

By taking subsequences, $\left(\omega_{n^{\prime}}\right)$ and $\left(\alpha_{n^{\prime}}\right)$ converge to the same measure $\mu$ and in particular one can find $\omega \in \omega(\underline{x}) \cap \operatorname{supp}(\mu)$ and $\alpha \in \alpha(\underline{y}) \cap \operatorname{supp}(\mu)$. Since $x \stackrel{W}{\rightarrow} \omega, \alpha \stackrel{W}{\rightarrow} y$ and $\omega \stackrel{W}{\rightarrow} \alpha$ by ergodicity of $\mu$, we obtain $x \stackrel{W}{\rightarrow} y$ for all $(x, y) \in \Omega(A, f): \Omega(A, f)$ is $W$-irreducible.

Proposition 25 shows that $\Sigma_{A, W}$ may contain invariant compact sets bigger than $\Omega(A, f)$ : it may contain heteroclinic orbits connecting two irreducible sets.

Proposition 16. Let $A: S^{1} \rightarrow \mathbb{R}$ be a $\alpha$-Hölder function.

(i) If $\Omega(A, f)$ is minimal then, for any sequence $\left(A_{n}\right)_{n \geq 0}$ of $\alpha$-Hölder functions converging to $A$ in the $\mathcal{C}^{\alpha}$-topology, the sequence of compact sets $\left(\Omega\left(A_{n}, f\right)\right)_{n \geq 0}$ converges to $\Omega(A, f)$ in the Hausdorff topology.

(ii) If $\Omega(A, f)$ is strictly ergodic, A satisfies the property of continuously varying support.

Proof. Step one. Using only the convergence of $\left(A_{n}\right)_{n \geq 0}$ to $A$ in the $\mathcal{C}^{0}$-topology, we show that $\left(m\left(A_{n}, f\right)\right)_{n \geq 0}$ converges to $m(A, f)$ and that, if $\mu_{n}$ is some maximizing measure for $\left(A_{n}, f\right)$, any weak limit of $\left(\mu_{n}\right)_{n \geq 0}$ is again a maximizing measure for $(A, f)$. Indeed, we have

$$
|m(A, f)-m(B, f)| \leq\|A-B\|_{0}
$$

for all $A, B$ and for any sub-sequence $\left(\mu_{n^{\prime}}\right)_{n^{\prime} \geq 0}$ converging to some measure $\mu$,

$$
\int A d \mu=\lim _{n^{\prime}} \int A_{n^{\prime}} d \mu_{n^{\prime}}=\lim _{n^{\prime}} m\left(A_{n^{\prime}}, f\right)=m(A, f) .
$$

Step two. Let $K_{n}=\Omega\left(A_{n}, f\right)$ and $K$ be a limit set in the Hausdorff topology of some sub-sequence $\left(K_{n^{\prime}}\right)_{n^{\prime} \geq 0}$. Thanks to Propositions 11 and 15(i), for each $n$, there exists a $\alpha$-Hölder sub-action $V_{n}$ for $\left(A_{n}, f\right)$ such that $K_{n}$ is included in the $V_{n}$-action set:

$$
A_{n}-m\left(A_{n}, f\right)=V_{n} \circ f-V_{n} \quad \text { on } K_{n} .
$$


We may assume that $V_{n}$ is normalized so that $V_{n}=0$ for some point in $K_{n}$. Since $\left(A_{n}\right)_{n}$ converges to $A$ in the $\mathcal{C}^{\alpha}$-topology, using Proposition 11 we may also assume that the $\alpha$-Hölder norm of $V_{n}$ is uniformly bounded. By Ascoli's theorem, we can extract a subsequence $\left(V_{n^{\prime}}\right)_{n^{\prime} \geq 0}$ which converges uniformly to an $\alpha$-Hölder function $V$. We have thus obtained a sub-action $V \in \mathcal{C}^{\alpha}$ for $(A, f)$ and a compact invariant set $K$ (i.e. $f(K)=K$ ) included in the $V$-action set. Since $\Omega(A, f)$ is $V$-irreducible by minimality, we know from Proposition 15(iii) that $K$ has to be included in $\Omega(A, f)$ and therefore has to be equal to $\Omega(A, f)$.

Step three. We assume now that $\Omega(A, f)$ is strictly ergodic and is equal to the support of the unique ergodic measure $\mu$. Let $\mu_{n}$ be some maximizing measure for $\left(A_{n}, f\right)$ and $K_{n}=\operatorname{supp}\left(\mu_{n}\right)$. Thanks to Proposition 15(ii), $K_{n}$ is included in $\Omega\left(A_{n}, f\right)$, and any accumulation set of $\left(K_{n}\right)_{n \geq 0}$ is contained in $\Omega(A, f)$ and equals $\Omega(A, f)$ by minimality. Any weak-accumulation measure has a support included in $\Omega(A, f)$ and is therefore equal to $\mu$ by unique ergodicity.

We are now able to prove the first part of Theorem 8 .

Proof of Theorem $8(i)$. We actually prove a little more. Let $\mathcal{Z}$ be the set of $\alpha$-Hölder potentials $A$ such that $\Omega(A, f)$ is minimal. We show that $\mathcal{Z}$ is generic in $\mathcal{C}^{\alpha}$. The set $\mathcal{R}$ of $\alpha$-Hölder potentials $A$ such that $\Omega(A, f)$ is uniquely ergodic is also generic according to Proposition 10. Therefore, $\mathcal{Z} \cap \mathcal{R}$ is generic and any $A \in \mathcal{Z} \cap \mathcal{R}$ satisfies the property of continuously varying support as is shown in Proposition 16(ii). For every $\epsilon>0$, we define

$$
\mathcal{Z}_{\epsilon}=\left\{A \in \mathcal{C}^{\alpha} \mid \exists n \geq 0, \forall y \in \Omega(A, f), \Omega(A, f) \subset \bigcup_{k=0}^{n} f^{-k}(B(y, \epsilon))\right\} .
$$

Let us first notice that $\mathcal{Z}=\cap_{\epsilon} \mathcal{Z}_{\epsilon}$. In order to show that $\mathcal{Z}$ is dense, for any $A \in \mathcal{C}^{\alpha}$, we choose some $K \subset \Omega(A, f)$ minimal. Then we can find $\phi \in \mathcal{C}^{\alpha}$ such that $\phi=0$ on $K, \phi>0$ everywhere outside $K$ and such that $A-\phi$ is $\mathcal{C}^{\alpha}$-close to $A$. Then $m(A-\phi, f)=m(A, f)$, any sub-action $V$ for $A$ is again a sub-action for $A-\phi$, the $V$-action set for $A-\phi$ is equal to $K$

$$
\{A-\phi-m=V \circ f-V\}=K
$$

and $\Omega(A-\phi, f)=K$ is minimal.

We now show that $\mathcal{Z}_{\epsilon}$ contains $\mathcal{Z}$ in its interior. Let $A \in \mathcal{Z}$, then there exists $n \geq 0$ such that

$$
\Omega(A, f) \subset \bigcup_{k=0}^{n} f^{-k}(B(y, \epsilon))
$$

for every $y \in \Omega(A, f)$. By compactness of $\Omega(A, f)$, there exists a neighborhood $U_{\epsilon}$ of $\Omega(A, f)$ such that

$$
U_{\epsilon} \subset \bigcup_{k=0}^{n} f^{-k}(B(y, \epsilon))
$$

for every $y \in U_{\epsilon}$. By Proposition 16(i), any $B$ close to $A$ (in the $\mathcal{C}^{\alpha}$-topology) satisfies $\Omega(B, f) \subset U_{\epsilon}$ and therefore is included in $\mathcal{Z}_{\epsilon}$. 
The strategy of the proof of Theorem 8(ii) is to find a periodic orbit well distributed and closed to $\Omega(A, f)$. We begin by proving a lemma of approximation in the $\mathcal{C}^{\alpha}$-topology for functions having a better regularity $\mathcal{C}^{\beta}, \beta>\alpha$.

Lemma 17. Let $0<\alpha<\beta<1$. For any $\beta$-Hölder functions $A$,

$$
\mathrm{Höl}_{\alpha}(A) \leq 2 \mathrm{Höl}_{\beta}(A)^{\alpha / \beta}\|A\|_{0}^{1-\alpha / \beta} \text {. }
$$

In particular, if $\left(A_{n}\right)$ is a sequence of $\beta$-hölder functions uniformly bounded in the $\mathcal{C}^{\beta}$. topology, if $\left(A_{n}\right)$ converges to zero in the $\mathcal{C}^{0}$-topology, then $\left(A_{n}\right)$ converges to zero in the $\mathcal{C}^{\alpha}$-topology.

Proof. The two estimates

$$
\begin{gathered}
|A(x)-A(y)| \leq \operatorname{Höl}_{\beta}(A) d(x, y)^{\beta} \leq \operatorname{Höl}_{\beta}(A) d(x, y)^{\beta-\alpha} d(x, y)^{\alpha}, \\
|A(x)-A(y)| \leq 2\|A\|_{0} \leq \frac{2\|A\|_{0}}{d(x, y)^{\alpha}} d(x, y)^{\alpha},
\end{gathered}
$$

show that $A$ is $\alpha$-Hölder with

$$
\operatorname{Höl}_{\alpha}(A) \leq 2 \sup _{x \neq y} \min \left(\frac{\|A\|_{0}}{d(x, y)^{\alpha}}, \operatorname{Höl}_{\beta}(A) d(x, y)^{\beta-\alpha}\right) .
$$

The minimum is reached at the intersection of the decreasing graph $t \mapsto\|A\|_{0} t^{-\alpha}$ and the increasing graph $t \mapsto \operatorname{Höl}_{\beta}(A) t^{\beta-\alpha}$.

Although the following is a very standard lemma, we need a precise estimate of the shadowing constant in the proof of Lemma 19 and so we give a proof.

Lemma 18. Let $f$ be $\mathcal{C}^{1}$-expanding, $\lambda=\lambda(f)$ and $N$ be such that $\lambda^{N}>2$. Then for any $x \in S^{1}$ such that $d\left(x, f^{N}(x)\right)$ is less than $\frac{1}{4}$, there exists a periodic point $p$ of period $N$ such that

$$
\forall 0 \leq k \leq N, \quad d\left(f^{k}(p), f^{k}(x)\right) \leq \frac{\lambda^{N}}{\lambda^{N}-1} \frac{d\left(x, f^{N}(x)\right)}{\lambda^{N-k}} .
$$

Notice that $\lambda^{N} /\left(\lambda^{N}-1\right) \rightarrow 1$ when $N \rightarrow+\infty$.

Proof. We call $x_{1}=f^{N}(x)$ and $x_{0}=x$. We assume $x_{1} \neq x_{0}$ (otherwise we choose $p=x_{0}$ ) and we denote by $] a, b\left[\right.$ the smallest unordered interval joining $a$ and $b$. Let $x_{0}^{\prime}$ be the closest pre-image of $x_{1}, f^{N}\left(x_{0}^{\prime}\right)=x_{1}$, such that $f^{N}$ restricted to $] x_{0}^{\prime}, x_{0}[$ is an homeomorphism onto $S^{1} \backslash\left\{x_{1}\right\}$ and such that $] x_{0}^{\prime}, x_{0}$ [ is disjoint from ] $x_{0}, x_{1}$ [ (we use the fact that $\lambda^{N}>2$ ). Let $x_{-1}$ be the unique pre-image of $x_{0}$ in $] x_{0}^{\prime}, x_{0}$ [ by the map $f^{N}$, then $\left.f^{N}(] x_{-1}, x_{0}[)=\right] x_{0}, x_{1}\left[\right.$ (we use the fact that $f^{N}$ preserves the orientation). By induction we obtain a sequence $\left(x_{-k}\right)$ of points $] x_{0}^{\prime}, x_{0}$ [ such that (]$x_{-k}, x_{-k+1}[)$ are pairwise disjoint and such that $f^{N}$ maps $] x_{-k-1}, x_{-k}$ [ onto $] x_{-k}, x_{-k+1}\left[\right.$. The point $p$, limit of $\left(x_{-k}\right)$ is therefore a periodic point of period $N$ and the length of the interval $] p, x_{1}[$ is bounded by

$$
d\left(x_{0}, x_{1}\right)\left[1+\frac{1}{\lambda^{N}}+\frac{1}{\lambda^{2 N}}+\cdots\right] \leq \frac{\lambda^{N}}{\lambda^{N}-1} d\left(x_{0}, x_{1}\right) .
$$

In particular, this length is bounded by $\frac{1}{2}, f^{N}$ maps $] p, x_{0}[$ onto $] p, x_{1}[$ and

$$
\forall 0 \leq k \leq N, \quad d\left(f^{k}(p), f^{k}\left(x_{0}\right)\right) \leq \frac{1}{\lambda^{N-k}} d\left(p, x_{1}\right) .
$$

The two inequalities combined end the proof. 
LEMMA 19. Let $\Omega$ be an invariant compact set, $f(\Omega)=\Omega$, without periodic orbit. Let $\eta=\frac{1}{2}(1-1 / \lambda)$. Then for any $\epsilon>0$ sufficiently small, there exists a periodic orbit $\left\{p, f(p), \ldots, f^{N}(p)=p\right\}$ such that

$$
d\left(f^{i}(p), \Omega\right)<\epsilon \quad \text { and } \quad d\left(f^{i}(p), f^{j}(p)\right)>\eta \epsilon,
$$

for all $0 \leq i<j<N$.

Proof. For each $K>0$ we define

$$
d(K)=\min \left\{d\left(f^{i}(x), f^{j}(x)\right) \mid x \in \Omega, 0 \leq i<j \leq K\right\} .
$$

Since no periodic orbit belongs to $\Omega, d(K)>0$ and tends to zero when $K$ tends to infinity. We now choose $K$ so that $\lambda^{K}>5$ and

$$
1-\frac{\lambda^{K}}{\lambda^{K}-1}\left(\frac{1}{\lambda}+\frac{1}{\lambda^{K}}\right)>\eta .
$$

Let $\epsilon$ be small enough such that $\epsilon<\frac{1}{3} d(K)$. We first exhibit an almost closed orbit $\left\{x, f(x), \ldots, f^{N}(x)\right\}$ in $\Omega$ satisfying

$$
d\left(x, f^{N}(x)\right)<\epsilon \quad \text { and } \quad d\left(f^{i}(x), f^{j}(x)\right) \geq \epsilon
$$

for all $0 \leq i<j \leq N$ except $i=0$ and $j=N$. This can be achieved by choosing an orbit $\left\{y, f(y), \ldots, f^{n}(y)\right\}$ in $\Omega$ such that $d\left(y, f^{n}(y)\right)<\epsilon$ and a sub-orbit $\left\{x, f(x), \ldots, f^{N}(x)\right\}$ with the property that $d\left(x, f^{N}(x)\right)<\epsilon$ and $N$ is minimal $(x$ is a point in the orbit of $y$ ). Since $\epsilon<d(K), N$ has to be bigger than $K$. By the shadowing lemma, Lemma 18, there exists a periodic point $p$ of period $N$ such that

$$
d\left(f^{i}(p), f^{i}(x)\right)<\frac{\lambda^{N}}{\lambda^{N}-1} \frac{\epsilon}{\lambda^{N-i}},
$$

for all $0 \leq i \leq N$. We now show that $p$ is the good candidate.

For $0 \leq i<j \leq N-K$,

$$
d\left(f^{i}(p), f^{i}(x)\right)<\frac{\epsilon}{4}, \quad d\left(f^{j}(p), f^{j}(x)\right)<\frac{\epsilon}{4}, \quad d\left(f^{i}(x), f^{j}(x)\right) \geq \epsilon
$$

and we obtain $d\left(f^{i}(p), f^{j}(p)\right)>\frac{1}{2} \epsilon>\eta \epsilon$.

For $N-K \leq i<j \leq N$,

$$
d\left(f^{i}(p), f^{i}(x)\right)<\epsilon, \quad d\left(f^{j}(p), f^{j}(x)\right)<\epsilon, \quad d\left(f^{i}(x), f^{j}(x)\right) \geq d(K)>3 \epsilon
$$

and we obtain in this case $d\left(f^{i}(p), f^{j}(p)\right)>\epsilon>\eta \epsilon$.

For $0 \leq i<N-K<j<N, d\left(f^{i}(x), f^{j}(x)\right) \geq \epsilon$ and

$$
d\left(f^{i}(p), f^{i}(x)\right)<\frac{\lambda^{K}}{\lambda^{K}-1} \frac{\epsilon}{\lambda^{K}}, \quad d\left(f^{j}(p), f^{j}(x)\right)<\frac{\lambda^{K}}{\lambda^{K}-1} \frac{\epsilon}{\lambda},
$$

and we obtain

$$
d\left(f^{i}(p), f^{j}(p)\right)>\epsilon\left(1-\frac{\lambda^{K}}{\lambda^{K}-1}\left(\frac{1}{\lambda}+\frac{1}{\lambda^{K}}\right)\right)>\eta \epsilon .
$$


Proof of Theorem $8(i i)$. Let $\mathcal{G}$ be the set of functions $A \in \mathcal{C}^{\alpha}$ having a unique maximizing measure supported on a periodic orbit and satisfying the property of continuously varying support. We first show that $\mathcal{G}$ is open in $\mathcal{C}^{\alpha}$. Let $A_{0} \in \mathcal{G}$ and $\mu_{0}=n^{-1} \sum_{k=0}^{n-1} \delta_{f^{k}}(p)$ be the unique maximizing measure supported on a periodic orbit $p$. By the property of continuously varying support, there exist a neighborhood $\mathcal{N} \operatorname{of} \operatorname{orb}(p)$ and a neighborhood $\mathcal{U}$ in $\mathcal{C}^{\alpha}$ of $A_{0}$ such that, for any $A \in \mathcal{U}$ and any $\mu \in \mathcal{M}(A, f)$, the support of $\mu$ is included in $\mathcal{N}$. By the standard shadowing lemma, $\mu=\mu_{0}$ is the only invariant measure close to $\operatorname{orb}(p)$. Then $\mathcal{U} \subset \mathcal{G}$.

We now show that $\mathcal{G}_{+}=\mathcal{G} \cap \mathcal{C}^{\alpha+}$ is dense in $\mathcal{C}^{\alpha+}$. Let $A_{1} \in \mathcal{C}^{\alpha+}$, then $A_{1} \in \mathcal{C}^{\beta}$, for some $\alpha<\beta<1$. We look for some $A=A_{1}+\phi, \phi \in \mathcal{C}^{\beta}$, with small $\|\cdot\|_{\alpha}$-norm such that $\Omega(A, f)$ is reduced to a single periodic orbit. From Proposition 16(ii), we know that $A$ will satisfy the property of continuous varying support. We actually prove the existence of a sequence $\left(\phi_{\epsilon}\right)$ of $\mathcal{C}^{\beta}$-functions having a $\beta$-norm, uniformly bounded and converging to zero in the uniform topology when $\epsilon$ goes to zero.

From Propositions 11 and 15(i), we can write

$$
A_{1}=m\left(A_{1}, f\right)+V_{1} \circ f-V_{1}-R_{1}
$$

where $V_{1}, R_{1} \in \mathcal{C}^{\beta}, R_{1} \geq 0$ and $R_{1}=0$ on $\Omega\left(A_{1}, f\right)$. Let $m\left(A_{1}, f\right)=m_{1}$.

We first construct some $\phi_{\epsilon} \in \mathcal{C}^{\beta}, \phi_{\epsilon} \geq 0$, with small $\|\cdot\|_{\alpha}$-norm such that $A_{1}+\phi_{\epsilon}$ admits a maximizing measure supported on a periodic orbit. If $\Omega(A, f)$ already contains a periodic orbit, we choose $\phi_{\epsilon}=0$. If not, from Lemma 19 , for any $\epsilon>0$, there exists a periodic orbit $\left\{p, \ldots, f^{N}(p)=p\right\}$ satisfying $d\left(q, \Omega\left(A_{1}, f\right)\right)<\epsilon$ and $d\left(q, q^{\prime}\right)>\eta \epsilon$ for any $q, q^{\prime} \in \operatorname{orb}(p), q \neq q^{\prime}$, where $\eta=\frac{1}{2}(1-1 / \lambda)$. We note that

$$
C_{1}=\mathrm{Höl}_{\beta}\left(R_{1}\right) \text {. }
$$

Since $R_{1}=0$ on $\Omega\left(A_{1}, f\right)$ and $R_{1}(q) \leq C_{1} d\left(q, \Omega\left(A_{1}, f\right)\right)^{\beta}$, we obtain for all $q \in \operatorname{orb}(p)$

$$
\epsilon>d\left(q, \Omega\left(A_{1}, f\right)\right) \geq\left(\frac{R_{1}(q)}{C_{1}}\right)^{1 / \beta} .
$$

We now define for each $q$ on the orbit a localized function $\phi_{\epsilon, q}$. The functions $\left\{\phi_{\epsilon, q}\right\}_{q \in \operatorname{orb}(p)}$ have disjoint support; they satisfy $0 \leq \phi_{\epsilon, q} \leq R_{1}$ everywhere and $\phi_{1, q}(q)=$ $R_{1}(q)$ for all $q \in \operatorname{orb}(p)$, by the following formula

$$
\phi_{\epsilon, q}(x)=\left[R_{1}(q)-D_{1} d(x, q)^{\beta}\right]^{+}
$$

where $D_{1}=(2 / \eta)^{\beta} C_{1}$. Clearly $D_{1} \geq C_{1}$ and by the Hölder property of $R_{1}$ we have $\phi_{\epsilon, q} \leq R_{1}$. The support of each $\phi_{\epsilon, q}$ is included in the ball of center $q$ and radius

$$
\left[\frac{R_{1}(q)}{D_{1}}\right]^{1 / \beta}=\left[\frac{R_{1}(q)}{C_{1}}\right]^{1 / \beta} \frac{\eta}{2}<\frac{1}{2} \epsilon \eta
$$

which shows that $\left\{\phi_{\epsilon, q}\right\}_{q \in \operatorname{orb}(p)}$ have disjoint support.

On the one hand, the $\mathrm{Höl}_{\beta}$-semi-norm of $\phi_{\epsilon, q}$ is uniformly bounded, independently of $\epsilon$,

$$
\operatorname{Höl}_{\beta}\left(\phi_{\epsilon, q}\right) \leq C_{1}+D_{1} \leq 2\left(\frac{2}{\eta}\right)^{\beta} C_{1}
$$

(we have used $\left|a^{+}-b^{+}\right| \leq|a-b|,\left|a^{\beta}-b^{\beta}\right| \leq|a-b|^{\beta}$ when $\beta<1$ ). 
On the other hand, the $\|\cdot\|_{0}$-norm of $\phi_{\epsilon, q}$ tends to zero when $\epsilon$ tends to zero,

$$
\left\|\phi_{\epsilon, q}\right\|_{0} \leq R_{1}(q) \leq C_{1} \epsilon^{\beta}
$$

We have thus obtained a non-negative function

$$
\phi_{\epsilon}=\sum_{q \in \operatorname{orb}(p)} \phi_{\epsilon, q}
$$

satisfying $\left\|\phi_{\epsilon}\right\|_{0} \leq C_{1} \epsilon^{\beta}$, Höl ${ }_{\beta}\left(\phi_{\epsilon}\right) \leq 4(2 / \eta)^{\beta} C_{1}, 0 \leq \phi_{\epsilon} \leq R_{1}$ everywhere and $\phi_{\epsilon}(q)=R_{1}(q)$ for all points $q \in \operatorname{orb}(p)$. By using Lemma 17, $\phi_{\epsilon} \in \mathcal{C}^{\beta}$ has small $\|\cdot\|_{\alpha}$-norm. Moreover, $m\left(A_{1}+\phi_{\epsilon}, f\right)=m_{1}$ and $\Omega\left(A_{1}+\phi_{\epsilon}, f\right)$ contains both $\Omega\left(A_{1}, f\right)$ and $\operatorname{orb}(p)$. Indeed

$$
A_{1}+\phi_{\epsilon}=V_{1} \circ f-V_{1}-R_{1}+\phi_{\epsilon}+m_{1} \leq V_{1} \circ f-V_{1}+m_{1} .
$$

If $x \in \Omega\left(A_{1}, f\right), \epsilon>0$ and $z, n>0$ have been chosen such that $d(x, z)<\epsilon$, $d\left(x, f^{n}(z)\right)<\epsilon$ and $\sum_{k=0}^{n-1} R \circ f^{k}(z)<\epsilon$, then $\sum_{k=0}^{n-1} \phi_{\epsilon} \circ f^{k}(z)<\epsilon$ and we have shown that $x \in \Omega\left(A_{1}+\phi_{\epsilon}, f\right)$. Actually, although we do not need it, we can show that

$$
\Omega\left(A_{1}+\phi_{\epsilon}, f\right)=\Omega(A, f) \cup \operatorname{orb}(p) .
$$

We now choose $\psi_{\epsilon} \geq 0, \psi_{\epsilon} \in \mathcal{C}^{\beta}$, with small $\|\cdot\|_{\beta}$-norm such that $\psi_{\epsilon}=0$ on orb $(p)$ and $\psi_{\epsilon}>0$ elsewhere. Then $A=A_{1}+\phi_{\epsilon}-\psi_{\epsilon} \in \mathcal{C}^{\beta}$ is close to $A_{0}$ in the $\mathcal{C}^{\alpha}$-topology and admits a unique maximizing measure supported on $\operatorname{orb}(p)$ :

$$
\mu=\frac{1}{\# \operatorname{orb}(p)} \sum_{q \in \operatorname{orb}(p)} \delta_{q} .
$$

Proof of Theorem $8($ iii $)$. We actually give a criterion to decide whether $\Omega(A, f)$ can stay stably a finite union of periodic points. The proof of Theorem 8 is a direct consequence of the following proposition.

Proposition 20. Let $f$ be a $\mathcal{C}^{1}$-expanding map, $0<\alpha<\beta$, and $A$ be a $\beta$-Hölder function. Then one of the following cases occurs.

(i) $A$ is a limit in the $\mathcal{C}^{\alpha}$-topology of $\mathcal{C}^{\beta}$-functions $\left(A_{n}\right)$, uniformly bounded in the $\mathcal{C}^{\beta}$-topology, having a unique maximizing measure $\mu_{n}$ whose support is strictly ergodic and has positive topological entropy.

(ii) $\quad \Omega(A, f)$ is a finite union of periodic points and there exists a $\mathcal{C}^{\beta}$ neighborhood $\mathcal{U}$ of A such that, for all $B \in \mathcal{U}, \Omega(B, f) \subset \Omega(A, f)$.

Before proving Proposition 20, we need the following lemma.

LEMMA 21. Let $K$ be a compact set (not necessarily invariant). Then $K$ admits a basis of closed neighborhoods $\mathcal{U}$ such that

$$
\Lambda_{\mathcal{U}}=\bigcap_{n \geq 0} f^{-n}(\mathcal{U})
$$

possesses the following property: 
(i) either $\Lambda_{\mathcal{U}}$ has positive entropy and $\Lambda_{\mathcal{U}}$ contains a strictly ergodic invariant compact set of positive entropy; or

(ii) $\Lambda_{\mathcal{U}}$ has zero entropy and the set of periodic points belonging to $\Lambda_{\mathcal{U}}$ is finite.

Proof. We first assume that $K$ is a compact set of the one-sided full shift $\mathcal{B}^{+}(D)$. Let $\mathcal{V}$ be an open set containing $K$ and $C_{1}, \ldots, C_{N}$, a finite union of cylinders covering $\mathcal{B}^{+}(D) \backslash \mathcal{V}$ and disjoint from $K$. We may assume the cylinders have all the same length. Let $\mathcal{U}=$ $\mathcal{B}^{+}(D) \backslash\left(C_{1} \cup \cdots \cup C_{N}\right)$. Then $\mathcal{U}$ is a closed neighborhood of $K$ and

$$
\Lambda_{\mathcal{U}}=\left\{x \in \mathcal{B}^{+}(D) \mid \forall i=1, \ldots, N, \forall n \geq 0, \sigma^{n}(x) \notin C_{i}\right\}
$$

is topologically conjugate to a one-sided Markov chain. If the entropy of $\Lambda_{\mathcal{U}}$ is equal to zero, the set of periodic points in $\Lambda_{\mathcal{U}}$ is finite. If the entropy of $\Lambda_{\mathcal{U}}$ is positive, we use Grillenberger's theorems [10] and [11] to construct a strictly ergodic compact set of positive entropy inside $\Lambda_{\mathcal{U}}$.

In the general case, let $K^{\prime}$ be a compact set of $S^{1}$ and $\mathcal{V}^{\prime}$ a neighborhood of $K^{\prime}$. We denote by $\pi: \mathcal{B}^{+}(D) \rightarrow S^{1}$ the canonical extension of $S^{1}$. Let $K=\pi^{-1}\left(K^{\prime}\right)$, $\mathcal{V}=\pi^{-1}\left(\mathcal{V}^{\prime}\right), C_{1} \ldots C_{N}, \mathcal{U}$ as before and $\mathcal{U}^{\prime}=\pi(\mathcal{U})$. Then $\mathcal{U}^{\prime}$ is a closed neighborhood of $K^{\prime}\left(\pi\left(C_{i}\right)\right.$ is disjoint from $\left.K^{\prime}\right)$ included in $\mathcal{V}^{\prime}$. Moreover, $\pi\left(\Lambda_{\mathcal{U}}\right) \subset \Lambda_{\mathcal{U}^{\prime}}$ and a point $x$ in $\Lambda_{\mathcal{U}^{\prime}}$ which does not belong to $\pi\left(\Lambda_{\mathcal{U}}\right)$ is necessarily a pre-image of one. Modulo a countable set in $\Lambda_{\mathcal{U}}$ and in $\Lambda_{\mathcal{U}^{\prime}}, \pi$ is a bijection, $\Lambda_{\mathcal{U}}, \Lambda_{\mathcal{U}^{\prime}}$ have the same topological entropy and the set of periodic points of $\Lambda_{\mathcal{U}^{\prime}}$ (except maybe one) is equal to the projection of the set of periodic points of $\Lambda_{\mathcal{U}}$.

Finally, if $L$ is a strictly ergodic invariant compact set, $\pi(L)$ is also strictly ergodic and $L$ and $\pi(L)$ have the same topological entropy.

Proof of Proposition 20. Step one. Assume there exists a $\mathcal{C}^{\beta}$ sub-action $V$ such that, for any closed neighborhood $\mathcal{U}$ of the $V$-action set

$$
K=\{A-m=V \circ f-V\}
$$

the invariant set $\Lambda_{\mathcal{U}}$ has positive topological entropy. As in the proof of Theorem 8(ii), we start by writing the cohomological equation for the sub-action $V$,

$$
A-m=V \circ f-V-R
$$

where $m=m(A, f)$ and $R$ is a non-negative $\mathcal{C}^{\beta}$-function which is equal to zero on $K$. We want to find a sequence of non-negative $\mathcal{C}^{\beta}$-functions $\left(\phi_{n}\right)$ converging to zero in the uniform topology and uniformly bounded in the $\mathcal{C}^{\beta}$-topology such that $V$ is still a subaction of $A+\phi_{n}$ and the $V$-action set of $A+\phi_{n}$ contains a neighborhood of $K$. We define

$$
\phi_{n}=\min \left(R, \frac{1}{n}\right)
$$

Then $0 \leq \phi_{n} \leq R, A+\phi_{n}-m \leq V \circ f-V$ and $\phi_{n}$ equals $R$ on a neighborhood $\mathcal{U}_{n}$ of $K$ (notice that $\left.m(A, f)=m\left(A+\phi_{n}, f\right)\right)$. By using Lemma $21, \mathcal{U}_{n}$ contains a strictly ergodic compact invariant set $L_{n}$ of positive topological entropy. We finally choose $\psi_{n}$ non-negative, $\psi_{n}=0$ on $L_{n}$ and $\psi_{n}>0$ on $S^{1} \backslash L_{n}$ with small $\|\cdot\|_{\beta}$-norm. We have just 
proved that $A_{n}=A+\phi_{n}-\psi_{n}$ converges to $A$ uniformly with bounded $\mathcal{C}^{\beta}$-norm and that each $\Omega\left(A_{n}, f\right)$ is strictly ergodic and has positive entropy.

Step two. Assume that for every $\mathcal{C}^{\beta}$ sub-action $V$ there exists a neighborhood $\mathcal{U}$ of $K$ such that $\Lambda_{\mathcal{U}}$ has zero entropy.

We first show that $\Omega(A, f)$ is a finite union of periodic points. Let $V$ be any sub-action. Then, by Lemma 21 , there exists a neighborhood $\mathcal{U}$ of $K$ such that $\Lambda_{\mathcal{U}}$ contains only a finite number of periodic points. If a periodic point belongs to $K$, it actually belongs to $\Omega(A, f)$. We can therefore choose $\mathcal{U}$ so that all periodic points in $\Lambda_{\mathcal{U}}$ are in $\Omega(A, f)$. Conversely, if $x$ is a point in $\Omega(A, f)$, then $x$ is a limit of periodic points $z_{n}$ of period $p_{n}$ such that $S_{p_{n}}(A-m)\left(z_{n}\right)$ tends to zero. Then $S_{p_{n}} R\left(z_{n}\right)$ also tends to zero and the orbit of $z_{n}$ has to stay closer and closer to $K$. For $n$ large enough, $z_{n}$ belongs to $\Lambda_{\mathcal{U}}$ and $x$ is a periodic point.

Let $\left(B_{n}\right)$ be a sequence of $\mathcal{C}^{\beta}$ functions, with bounded $\mathcal{C}^{\beta}$-norm, converging to $A$ in the $\mathcal{C}^{\alpha}$-topology. We want to show that $\Omega\left(B_{n}, f\right) \subset \Omega(A, f)$ for $n$ sufficiently large. As in step two of the proof of Proposition 16, for each $B_{n}$ we choose a sub-action $V_{n}$ and we may assume (maybe by taking a sub-sequence) that $\left(V_{n}\right)$ converges to some sub-action $V$ of class $\mathcal{C}^{\beta}$ and that the $V_{n}$-action set $K_{n}$ converges to some compact set included in the $V$-action set. We choose a neighborhood $\mathcal{U}$ as before so that the only periodic points included in $\Lambda_{\mathcal{U}}$ are actually in $\Omega(A, f)$. For $n$ large enough, $\Omega\left(B_{n}, f\right) \subset K_{n} \subset \mathcal{U}$ and by the same argument as before $\Omega\left(B_{n}, f\right)$ has to be included into the closure of the set of periodic points of $\Lambda_{\mathcal{U}}$. That is, for large $n, \Omega\left(B_{n}, f\right) \subset \Omega(A, f)$.

The rest of this section is independent of Theorem 8 . The reader interested just in the minimization of Lyapunov measures can go directly to $\S 3$. We first show how to construct other sub-actions from a function $S_{A}(x, y)$ that we call the action potential. We then define the notion of a $V$-heteroclinic orbit which is stronger than the notion of $V$-connection and apply it to the case where there is a finite number of irreducible sets.

Definition 22. Let $(x, y) \in S^{1}$ and $A: S^{1} \rightarrow \mathbb{R}$. We call the action potential of $A$ from $x$ to $y$ the following quantity:

$$
S_{A}(x, y)=\lim _{\epsilon \rightarrow 0} \sup \left\{S_{n}(A-m)\left(x^{\prime}\right) \mid n \geq 1, d\left(x^{\prime}, x\right)<\epsilon, f^{n}\left(x^{\prime}\right)=y\right\}
$$

(notice that we take the supremum over all paths of length $n \geq 1$ ending at $y$ and beginning within $\epsilon$ of $x$ ).

As in the definition of $V_{A}$ in Proposition 11, $S_{A}(x, y)$ is uniformly bounded from above but it may happen that $S_{A}(x, y)=-\infty$. The interesting case takes place when $x \in \Omega(A, f)$.

Proposition 23. Let $f$ be a $\mathcal{C}^{1}$-expanding map and $A$ be a $\mathcal{C}^{\alpha}$-function. Then the action potential of A satisfies the following properties:

(i) for any sub-action $V$, for any $x, y \in S^{1}, S_{A}(x, y) \leq V(y)-V(x)$ and $x \stackrel{V}{\rightarrow} y$ if and only if $S_{A}(x, y)=V(y)-V(x)$;

(ii) for any $x, y, z \in S^{1}, S_{A}(x, y)+S_{A}(y, z) \leq S_{A}(x, z), S_{A}(x, x) \leq 0$ and $S_{A}(x, x)=$ 0 if and only if $x \in \Omega(A, f)$;

(iii) for any $x \in \Omega(A, f), S_{A}(x, \cdot)$ is a $\mathcal{C}^{\alpha}$ sub-action (in particular, $S_{A}(x, y)>-\infty$ for any $\left.y \in S^{1}\right)$. 
Proof. (We only prove part (iii).) Let $x \in \Omega(A, f)$ be fixed.

Step one. We first show that $S_{A}(x, \cdot)$ never takes the value $-\infty$. Let us define an approximate action potential

$$
S_{A}^{\epsilon}(x, y)=\sup \left\{S_{n}(A-m)\left(x^{\prime}\right) \mid d\left(x, x^{\prime}\right)<\epsilon, f^{n}\left(x^{\prime}\right)=y, n \geq 1\right\}
$$

where the supremum is taken over all paths of length $n \geq 1$ starting within $\epsilon$ of $x$ and ending at $y$. Then $S_{A}(x, y)=\inf _{\epsilon} S_{A}^{\epsilon}(x, y)$. Let $\epsilon_{0}<\frac{1}{2}$ and $n_{0} \geq 1, x^{\prime}$ such that $d\left(x, x^{\prime}\right)<\epsilon_{0}, f^{n_{0}}\left(x^{\prime}\right)=y$ and $S_{n_{0}}(A-m)\left(x^{\prime}\right)$ is close to $S_{A}^{\epsilon}(x, y)$. Since $x$ belongs to $\Omega(A, f)$, for every $\epsilon>0$, there exist $n \geq 1$, large enough so that $\lambda^{-n} \epsilon_{0}<\epsilon / 2, z$ such that $d(z, x)<\epsilon / 2, f^{n}(z)=x$ and $\left|S_{n}(A-m)(z)\right|<\epsilon$. We choose $z^{\prime}$ in the same inverse branch of length $n$ as $z$ such that $f^{n}\left(z^{\prime}\right)=x^{\prime}$. Then $d\left(z, z^{\prime}\right) \leq \lambda^{-n} d\left(x, x^{\prime}\right)<\epsilon / 2$, $d\left(x, z^{\prime}\right)<\epsilon$ and

$$
\begin{gathered}
\left|S_{n}(A-m)(z)-S_{n}(A-m)\left(z^{\prime}\right)\right|<\frac{\operatorname{Höl}_{\alpha}(A)}{\lambda^{\alpha}-1} \epsilon_{0}, \\
S_{n_{0}+n}(A-m)\left(z^{\prime}\right) \geq S_{n_{0}}(A-m)\left(x^{\prime}\right)-\frac{\operatorname{Hö}_{\alpha}(A)}{\lambda^{\alpha}-1} \epsilon_{0}-\epsilon .
\end{gathered}
$$

By letting $\epsilon$ go to zero, we obtain

$$
S_{A}(x, y)>S_{A}^{\epsilon_{0}}(x, y)-\frac{\operatorname{Höl}_{\alpha}(A)}{\lambda^{\alpha}-1} \epsilon_{0} .
$$

This section shows in addition that, in the definition of $S_{A}^{\epsilon}(x, y), n$ can be as large as we want.

Step two. Let $(y, z) \in S^{1}$ such that $d(y, z)<\frac{1}{2}$. Let $\epsilon>0$ and $n \geq 1$ such that $\lambda^{-n}<\epsilon$. Then there exists $x^{\prime}$ such that $d\left(x, x^{\prime}\right)<\epsilon, f^{n}\left(x^{\prime}\right)=y$ and $S_{A}^{\epsilon}(x, y)<S_{n}(A-m)\left(x^{\prime}\right)+\epsilon$. Let $x^{\prime \prime}$ be the unique $n$-pre-image of $z$ in the same inverse branch as $x^{\prime}$. Then $d\left(x^{\prime} x^{\prime \prime}\right)<\epsilon$, $d\left(x, x^{\prime \prime}\right)<2 \epsilon$ and

$$
S_{n}(A-m)\left(x^{\prime}\right) \leq s_{n}(A-m)\left(x^{\prime \prime}\right)+\frac{\operatorname{Höl}_{\alpha}(A)}{\lambda^{\alpha}-1} d(y, z) .
$$

Combining all these inequalities we obtain

$$
\begin{aligned}
S_{A}(x, y) & \leq S_{A}^{\epsilon}(x, y) \\
& \leq S_{n}(A-m)\left(x^{\prime}\right)+\epsilon \\
& \leq S_{A}^{2 \epsilon}+\epsilon+\frac{\operatorname{Höl}_{\alpha}(A)}{\lambda^{\alpha}-1} d(y, z) \\
& \leq S_{A}(x, z)+\epsilon+\frac{\operatorname{Höl}_{\alpha}(A)}{\lambda^{\alpha}-1}[2 \epsilon+d(y, z)]
\end{aligned}
$$

and $S_{A}(x, \cdot)$ is $\mathcal{C}^{\alpha}$ with $\alpha$-Hölder constant $\operatorname{Höl}_{\alpha}(A) /\left(\lambda^{\alpha}-1\right)$.

Step three. We show that $S_{A}(x, \cdot)$ is a sub-action. For $\epsilon>0$ we have clearly

$$
S_{A}^{\epsilon}(x, y)+(A-m)(y) \leq S_{A}^{\epsilon}(x, f(y)) .
$$

By letting $\epsilon$ go to zero we obtain

$$
(A-m)(y) \leq S_{A}(x, f(y))-S_{A}(x, y) .
$$


From the first part of Proposition 23, we notice that, if an invariant compact set $K$ is irreducible for two sub-actions $V$ and $W$, then $V-W$ is constant on $K$. The last part of the proposition shows that, for any $x \in \Omega(A, f)$, there exists a sub-action $V(y)=S_{A}(x, y)$ such that any $y \in S^{1}$ is $V$-connected to $x(x \stackrel{V}{\rightarrow} y)$.

We now define a stronger notion of $V$-connection.

Definition 24. Let $V$ be a sub-action and $\Omega_{0}, \Omega_{1}$ be two disjoint $V$-irreducible compact invariants sets of $\Omega(A, f)$. We say that $\left(\Omega_{0}, \Omega_{1}\right)$ are strongly $V$-connected if there exists a complete orbit $\underline{x}=\left(x_{n}\right)_{n \in \mathbb{Z}}$ included in the $V$-action set such that $\alpha(\underline{x}) \subset \Omega_{0}$ and $\omega(\underline{x}) \subset \Omega_{1}$.

If $\Omega_{0}$ and $\Omega_{1}$ are periodic orbits of $\Omega(A, f)$ and strongly $V$-connected, such a complete orbit $\underline{x}$ satisfying $\alpha(\underline{x}) \subset \Omega_{0}$ and $\omega(\underline{x}) \subset \Omega_{1}$ could be called a heteroclinic orbit.

Proposition 25. Let $V$ be a sub-action and let us assume that $\Omega(A, f)$ is equal to a finite disjoint union of $V$-irreducible compact invariant sets $\left\{\Omega_{i}\right\}_{i=1}^{N}$. Then each $\Omega_{i}$ is a maximal $V$-irreducible invariant compact set and cannot be a disjoint union of two invariant compact sets. Let $i \neq j$. If $\left(\Omega_{i}, \Omega_{j}\right)$ are $V$-connected, then there exist a chain $\left(i_{0}, i_{1}, \ldots, i_{r}\right)$ of pairwise distinct indexes in $\{1, \ldots, N\}$ such that $i_{0}=i, i_{r}=j$ in each $\left(\Omega_{i_{k-1}}, \Omega_{i_{k}}\right)$ are strongly $V$-connected.

Proof. Step one. Let $K$ be an invariant compact set and assume that $K$ can be written as a disjoint union of two (not necessarily $V$-irreducible) compact invariant sets $K_{0}$ and $K_{1}$. We assume, moreover, that there exist $p_{0} \in K_{0}$ and $p_{1} \in K_{1}$ which are $V$-connected. We show that, if $U_{0}$ and $U_{1}$ are disjoint open sets containing $K_{0}$ and $K_{1}$, there exists $x_{0} \notin K_{0} \cup K_{1}$ and a complete orbit $\underline{x}$ included in the $V$-action set going through $x_{0}$ such that $\alpha(\underline{x}) \subset \bar{U}_{0}$ and

$$
p_{0} \stackrel{V}{\rightarrow} \alpha(\underline{x}) \stackrel{V}{\rightarrow} x_{0} \stackrel{V}{\rightarrow} \omega(\underline{x}) \stackrel{V}{\rightarrow} p_{1} .
$$

Since $\left(p_{0}, p_{1}\right)$ are $V$-connected, for every $\epsilon>0$, there exist $n_{\epsilon} \geq 1$ and $p_{\epsilon}$ such that $d\left(p_{0}, p_{\epsilon}\right)<\epsilon, f^{n_{\epsilon}}\left(p_{\epsilon}\right)=p_{1}$ and

$$
V\left(p_{1}\right)-V\left(p_{\epsilon}\right)-\epsilon \leq S_{n}(A-m)\left(p_{\epsilon}\right) \leq V\left(p_{1}\right)-V\left(p_{\epsilon}\right)
$$

Let us show, for all $0 \leq k<l \leq n$,

$$
V \circ f^{l}\left(p_{\epsilon}\right)-V \circ f^{k}\left(p_{\epsilon}\right)-\epsilon \leq S_{l-k}(A-m) \circ f^{k}\left(p_{\epsilon}\right)
$$

and

$$
S_{l-k}(A-m) \circ f^{k}\left(p_{\epsilon}\right) \leq V \circ f^{l}\left(p_{\epsilon}\right)-V \circ f^{k}\left(p_{\epsilon}\right) .
$$

On the one hand, because $V$ is a sub-action

$$
S_{l-k}(A-m) \circ f^{k}\left(p_{\epsilon}\right) \leq V \circ f^{l}\left(p_{\epsilon}\right)-V \circ f^{k}\left(p_{\epsilon}\right) .
$$

On the other hand,

$$
\begin{aligned}
V\left(p_{1}\right)-V\left(p_{\epsilon}\right)-\epsilon \leq & S_{n-l}(A-m) \circ f^{l}\left(p_{\epsilon}\right) \\
& +S_{l-k}(A-m) \circ f^{k}\left(p_{\epsilon}\right)+S_{k}(A-m)\left(p_{\epsilon}\right),
\end{aligned}
$$


and

$$
\begin{aligned}
S_{k}(A-m)\left(p_{\epsilon}\right) & \leq V \circ f^{k}\left(p_{\epsilon}\right)-V\left(p_{\epsilon}\right), \\
S_{n-l}(A-m) \circ f^{l}\left(p_{\epsilon}\right) & \leq V\left(p_{1}\right)-V \circ f^{l}\left(p_{\epsilon}\right) .
\end{aligned}
$$

Combining these inequalities we obtain

$$
V \circ f^{l}\left(p_{\epsilon}\right)-V \circ f^{k}\left(p_{\epsilon}\right)-\epsilon \leq S_{l-k}(A-m) \circ f^{k}\left(p_{\epsilon}\right) .
$$

Let $s_{\epsilon}$ be the first time the iterates of $p_{\epsilon}$ escape $U_{0}$, that is, $f^{k}\left(p_{\epsilon}\right) \in U_{0}$ for $k=$ $0,1, \ldots, s_{\epsilon}$ and $f^{s_{\epsilon}+1}\left(p_{\epsilon}\right) \notin U_{0}$. Let $x_{-k}(\epsilon)=f^{s_{\epsilon}-k}\left(p_{\epsilon}\right)$. By a procedure of diagonal extraction, we can find a subsequence of $\epsilon$ 's converging to zero such that each $x_{-k}(\epsilon)$ converges to some $x_{-k}$ (note that $s_{\epsilon} \rightarrow \infty$ when $\epsilon \rightarrow 0$ ). By construction, $x_{0} \in \bar{U}_{0} \backslash f^{-1}\left(U_{0}\right), x_{-k} \in \bar{U}_{0}$ for all $k=1,2, \ldots$ and the complete orbit $\underline{x}=\left(x_{n}\right)$ is included in the $V$-action set. Moreover, for any $n \in \mathbb{Z}, p_{0} \stackrel{V}{\rightarrow} x_{n} \stackrel{V}{\rightarrow} p_{1}$.

Step two. We show that a maximal (for the inclusion) $V$-irreducible compact invariant set $K$ cannot be equal to a disjoint union of two invariant compact sets $K_{0}$ and $K_{1}$. Otherwise, we choose $p_{0} \in K_{0}, p_{1} \in K_{1}$ and by assumption $\left(p_{0}, p_{1}\right)$ are $V$-connected in both directions. Thanks to step one there exist complete orbits $\underline{x}$ and $\underline{y}$ included in the $V$-action set such that $x$ and $y$ do not belong to $K$ and

$$
p_{0} \stackrel{V}{\rightarrow} \operatorname{orb}(\underline{x}) \stackrel{V}{\rightarrow} p_{1} \stackrel{V}{\rightarrow} \operatorname{orb}(\underline{y}) \stackrel{V}{\rightarrow} p_{0} .
$$

The set $K_{0} \cup \operatorname{orb}(\underline{x}) \cup K_{1} \cup \operatorname{orb}(\underline{y})$ is compact, invariant and $V$-irreducible, which contradicts the maximality of $K$.

Step three. We assume from now on that $\Omega(A, f)$ is equal to a disjoint union of irreducible compact invariant sets $\left\{\Omega_{i}\right\}_{i=1}^{N}$ and that $\Omega_{i}$ is $V$-connected to $\Omega_{j}$. Let $i_{0}=i$. There exists a complete orbit $\underline{x}_{i_{0}}$ not included in $\Omega(A, f)$ such that $\alpha\left(\underline{x}_{i_{0}}\right) \subset \Omega_{i_{0}}$ and $\omega\left(\underline{x}_{i_{0}}\right)$ is $V$-connected to $\Omega_{j}$ (we use the fact that $\alpha\left(\underline{x}_{i_{0}}\right)$ can be chosen in a neighborhood of $\Omega_{i_{0}}$ and has to be included in $\Omega(A, f)$ ). But $\omega\left(\underline{x}_{i_{0}}\right)$ is included in $\Omega(A, f)$ and has to be included into some $\Omega_{i_{1}}$ (thanks to step two $\omega\left(\underline{x}_{i_{0}}\right)$ cannot intersect two $\Omega_{i}$ ). Necessarily $i_{1} \neq i_{0}$, otherwise, by irreducibility of $\Omega_{i_{0}}$ we would have

$$
\alpha\left(\underline{x}_{i_{0}}\right) \stackrel{V}{\rightarrow} \underline{x}_{i_{0}}(0) \stackrel{V}{\rightarrow} \omega\left(\underline{x}_{i_{0}}\right) \stackrel{V}{\rightarrow} \alpha\left(\underline{x}_{i_{0}}\right)
$$

and $\underline{x}_{i_{0}}(0)$ would belong to $\Omega_{i_{0}}$. Either $i_{1}=j$ and we are done or we repeat the previous construction. There exists $\underline{x}_{i_{1}}$ such that $\alpha\left(\underline{x}_{i_{1}}\right) \subset \Omega_{i_{1}}, \omega\left(\underline{x}_{i_{1}}\right)$ is contained in some $\Omega_{i_{2}}$ and $\underline{x}_{i_{1}}(0) \notin \Omega(A, f)$. By irreducibility of $\Omega_{i_{0}}$ or $\Omega_{i_{1}}, i_{2}$ cannot be equal to $i_{0}$ or $i_{1}$. This process has to stop since the number of irreducible parts is finite.

\section{Lyapunov minimizing measures}

The idea of the proof of Theorem 2 is first to conjugate $f$ to a fixed map $T$ by preserving the same geometry of the orbits and second to transfer the smoothness of $f$ into two invariants $(A, \mu)$ where $A=-\ln f^{\prime}$ has pressure zero and $\mu$, the Lebesgue measure, is the unique equilibrium measure associated to $(A, f)$.

We first show in the following lemma that any maps $(f, g)$ in $\mathcal{F}_{\alpha}$ are conjugate by biHölder maps and that the Hölder exponent can be as close to 1 as we want, depending 
on the $\mathcal{C}^{1+\alpha}$-distance of $f$ and $g$. Notice that the composition of an $\alpha$-Hölder map with a $\beta$-Hölder map is $\alpha \beta$-Hölder. We use the notation $a \wedge b=\min (a, b)$ and $a \vee b=\max (a, b)$.

LEMMA 26. For simplicity we assume all maps in $\mathcal{F}_{\alpha}$ fix $1 \in S^{1}$.

(i) For any $\mathcal{C}^{1} f$ and $g$ in $\mathcal{F}_{\alpha}$, there exists a unique orientation-preserving homeomorphism $\theta=\theta_{f, g}$ such that $\theta(1)=1$ and $f \circ \theta=\theta \circ g$. The conjugating map is bi-Hölder:

$$
C^{-1 / \gamma} d(x, y)^{1 / \gamma} \leq d(\theta(x), \theta(y)) \leq C d(x, y)^{\gamma}, \quad \forall x, y \in S^{1}
$$

with Hölder exponent $\gamma=\gamma(f, g)$ and $\gamma$-Hölder norm $C=C(g)$ :

$$
\begin{gathered}
\gamma(f, g)=\inf \left\{\frac{\ln f^{\prime}(x)}{\ln g^{\prime}(y)} \wedge \frac{\ln g^{\prime}(x)}{\ln f^{\prime}(y)} \mid d(x, y) \leq \frac{\|\bar{f}-\bar{g}\|_{0}}{\lambda(f) \vee \lambda(g)-1}\right\} \\
\lambda(g)=\min _{x \in S^{1}} g^{\prime}(x), \quad C(g)=2 \max _{0 \leq i<D} d\left(z_{i}^{g}, z_{i+1}^{g}\right)^{-1} \exp \left(\frac{\mathrm{Höl}_{\alpha}\left(g^{\prime}\right)}{\lambda(g)^{\alpha}-1}\right)
\end{gathered}
$$

where $z_{0}^{g}, \ldots, z_{D-1}^{g}$ are the $D$ pre-images of 1 by $g$.

(ii) Let $T \in \mathcal{F}_{\alpha}$ be fixed. Then for any $f, g \in \mathcal{F}_{\alpha}, \theta_{f}=\theta_{f, T}$ and $\theta_{g}=\theta_{g, T}$ satisfy

$$
\begin{aligned}
\left\|\bar{\theta}_{f}-\bar{\theta}_{g}\right\|_{0} & \leq \frac{\|\bar{f}-\bar{g}\|_{0}}{\lambda(f) \vee \lambda(g)-1} \\
\left\|\left(\bar{\theta}_{f}\right)^{-1}-\left(\bar{\theta}_{g}\right)^{-1}\right\|_{0} & \leq C(T)\left\|\bar{\theta}_{f}-\bar{\theta}_{g}\right\|_{0}^{\gamma(T, f) \vee \gamma(T, g)}
\end{aligned}
$$

where $\bar{\theta}_{f}, \bar{\theta}_{g}: \mathbb{R} \rightarrow \mathbb{R}$ denote the lifts of $\theta_{f}, \theta_{g}$ with $\bar{\theta}_{f}(0)=\bar{\theta}_{g}(0)=0$.

Proof. Let $f \in \mathcal{F}_{\alpha}, z_{0}^{f}=1$ and $z_{1}^{f} \ldots z_{D-1}^{f}$ be the pre-images of 1 by $f$ ordered canonically on $S^{1} \backslash\{1\}$. More generally, we call $Z(f)$ the set of pre-images of 1 by $\left(f^{n}\right), n \geq 0$, and code the points in $Z(f)$ by points of the form $\left\{z_{i_{1} \ldots i_{n}}^{f}\right\}$ where $i_{n}=0,1, \ldots, D-1$. For instance, when $D=2$ the pre-images are ordered in the following way:

$$
1=z_{0 \ldots 000}^{f}, z_{0 \ldots 001}^{f}, z_{0 \ldots 010}^{f}, z_{0 \ldots 011}^{f}, z_{0 \ldots 100}^{f}, \ldots, z_{1 \ldots 111}^{f}<1 .
$$

Moreover, $Z(f)$ is dense in $S^{1}$, otherwise any non-empty connected component of $S^{1} \backslash Z(f)$ would be permuted without containing the point 1 and this would contradict the uniform growth of the length.

Since the conjugating map $\theta_{f, g}$ has to preserve the order of the pre-images, we have necessarily

$$
\theta_{f, g}\left(z_{i}^{g}\right)=z_{i}^{f}, \quad \forall i=i_{1} \ldots i_{n} .
$$

Then $\theta_{f, g}$ extends uniquely to a homeomorphism which conjugates $f$ and $g$. Let $\bar{f}$ be the lifting of $f$ fixing 0 . We denote by $f_{i}^{-1}:[0,1[\rightarrow[0,1[, i=0,1, \ldots, D-1$, the inverse branches of $\bar{f}$ :

$$
\bar{f} \circ f_{i}^{-1}(w)=w+i, \quad \forall w \in[0,1[.
$$

Then the points $\left(z_{i_{1} \ldots i_{n}}^{f}\right)$ on $S^{1}$ correspond to the points

$$
\bar{z}_{i_{1} \ldots i_{n}}^{f}=f_{i_{1}}^{-1} \circ \cdots \circ f_{i_{n}}^{-1}(0)
$$


on $[0,1[$. We first prove that for any $w$ on $[0,1[, n \geq 1$ :

$$
\left|w_{i_{1} \ldots i_{n}}^{f}-w_{i_{1} \ldots i_{n}}^{g}\right| \leq \frac{\|\bar{f}-\bar{g}\|_{0}}{\lambda(f) \vee \lambda(g)-1}
$$

where $w_{i_{1} \ldots i_{n}}^{f}=f_{i_{1}}^{-1} \circ \cdots \circ f_{i_{n}}^{-1}(w)$. By induction

$$
\begin{aligned}
\left|w_{j i_{1} \ldots i_{n}}^{f}-w_{j i_{1} \ldots i_{n}}^{g}\right| & \leq\left|f_{j}^{-1}\left(w_{i_{1} \ldots i_{n}}^{f}\right)-f_{j}^{-1}\left(w_{i_{1} \ldots i_{n}}^{g}\right)\right|+\left|f_{j}^{-1}\left(w_{i_{1} \ldots i_{n}}^{g}\right)-g_{j}^{-1}\left(w_{i_{1} \ldots i_{n}}^{g}\right)\right| \\
& \leq \frac{1}{\lambda(f)}\left[\left|w_{i_{1} \ldots i_{n}}^{f}-w_{i_{1} \ldots i_{n}}^{g}\right|+\|\bar{f}-\bar{g}\|_{0}\right] .
\end{aligned}
$$

(The second term is obtained using the estimate

$$
\left|\bar{f}^{-1}(u)-\bar{g}^{-1}(u)\right|=\left|\bar{f}^{-1} \circ \bar{g} \circ \bar{g}^{-1}(u)-\bar{f}^{-1} \circ \bar{f} \circ \bar{g}^{-1}(u)\right|
$$

for any $u \in[0, D[$.) In particular, this proves

$$
\begin{aligned}
\left\|\bar{\theta}_{f, T}-\bar{\theta}_{g, T}\right\|_{0} & \leq \sup _{i=i_{1} \ldots i_{n}}\left|\bar{\theta}_{f, T}\left(\bar{z}_{i}^{T}\right)-\bar{\theta}_{g, T}\left(\bar{z}_{i}^{T}\right)\right|=\sup _{i=i_{1} \ldots i_{n}}\left|\bar{z}_{i}^{f}-\bar{z}_{i}^{g}\right| \\
& \leq \frac{\|\bar{f}-\bar{g}\|_{0}}{\lambda(f) \vee \lambda(g)-1} .
\end{aligned}
$$

Assuming that $\theta_{f}=\theta_{f, T}$ is $\beta$-Hölder, we obtain

$$
\begin{aligned}
\left\|\bar{\theta}_{f}^{-1}-\bar{\theta}_{g}^{-1}\right\|_{0} & =\left\|\bar{\theta}_{f}^{-1} \circ \bar{\theta}_{g} \circ \bar{\theta}_{g}^{-1}-\bar{\theta}_{f}-1 \circ \bar{\theta}_{f} \circ \bar{\theta}_{g}^{-1}\right\|_{0} \\
& \leq \operatorname{Höl}_{\gamma}\left(\bar{\theta}_{f}^{-1}\right)\left\|\bar{\theta}_{g}-\bar{\theta}_{f}\right\|_{0}^{\gamma} .
\end{aligned}
$$

We now prove that $\theta=\theta_{f, g}$ is Hölder. We begin by proving that, for all $n \geq 1$, for any consecutive pre-images of order $n$,

$$
\bar{z}_{i}^{g}=g_{i_{1}}^{-1} \circ \cdots \circ g_{i_{n}}^{-1}(0), \quad \bar{z}_{i+1}^{g}=g_{i_{1}}^{-1} \circ \cdots \circ g_{i_{n}}^{-1}(1),
$$

we have $\bar{z}_{i}^{f}=\bar{\theta}\left(\bar{z}_{i}^{g}\right)$ and $\left|\bar{z}_{i}^{f}-\bar{z}_{i+1}^{f}\right| \leq\left|\bar{z}_{i}^{g}-\bar{z}_{i+1}^{g}\right|^{\beta}$. Indeed

$$
\left|\bar{z}_{i}^{f}-\bar{z}_{i+1}^{f}\right|=\int_{0}^{1} \prod_{k=1}^{n}\left[\frac{D}{D w} f_{i_{k}}^{-1}\right]\left(w_{i_{k+1} \cdots i_{n}}^{f}\right) d w .
$$

Since $\left|w_{i_{k} \ldots i_{n}}^{f}-w_{i_{k} \ldots i_{n}}^{g}\right| \leq\|\bar{f}-\bar{g}\|_{0} /[\lambda(f) \vee \lambda(g)-1]$, we obtain by definition of $\gamma(f, g)$

$$
\left|\bar{z}_{i}^{f}-\bar{z}_{i+1}^{f}\right| \leq \int_{0}^{1} \prod_{k=1}^{n}\left[\frac{D}{D w} g_{i_{k}}^{-1}\right]^{\gamma}\left(w_{i_{k+1} \cdots i_{n}}^{g}\right) d w \leq\left|\bar{z}_{i}^{g}-\bar{z}_{i+1}^{g}\right|^{\gamma} .
$$

(We use Jensen's inequality to extract the power $\gamma$ outside the integral since $\gamma(f, g)<1$ for any $f \neq g$.) Let $\bar{z}_{i}^{g}$ and $\bar{z}_{i+1}^{g}\left(i=i_{1} \ldots i_{n}\right)$ be two consecutive pre-images of order $n$, then $\left[\bar{z}_{i}^{g}, \bar{z}_{i+1}^{g}\right.$ [ contains $D$ pre-images of order $n+1$ that we call $\bar{z}_{i j}^{g}$, where $i j=i_{i} \ldots i_{n} j$ and $j=0,1, \ldots, D-1$. We show that for any $j=0, \ldots, D-1$,

$$
\left|\bar{z}_{i}^{g}-\bar{z}_{i+1}^{g}\right| \leq \frac{1}{2} C(g)\left|\bar{z}_{i j}^{g}-\bar{z}_{i j+1}^{g}\right| .
$$


where $C(g)$ is given in the above lemma. It is enough to show that the distortion of $g_{i_{1}}^{-1} \circ \cdots \circ g_{i_{n}}^{-1}$ is uniformly bounded by $\exp \left(\operatorname{Höl}_{\alpha}\left(g^{\prime}\right) /\left(\lambda(g)^{\alpha}-1\right)\right)$, that is

$$
\left|\frac{\frac{d}{d w} g_{i_{1}}^{-1} \circ \cdots \circ g_{i_{n}}^{-1}(u)}{\frac{d}{d w} g_{i_{1}}^{-1} \circ \cdots \circ g_{i_{n}}^{-1}(v)}\right|=\prod_{k=1}^{n}\left|\frac{g^{\prime}\left(v_{i_{k} \cdots i_{n}}^{g}\right)}{g^{\prime}\left(u_{i_{k} \cdots i_{n}}^{g}\right)}\right| \leq \exp \sum_{k=1}^{n}\left|g^{\prime}\left(v_{i_{k} \cdots i_{n}}^{g}\right)-g^{\prime}\left(u_{i_{k} \cdots i_{n}}^{g}\right)\right|
$$

is bounded by $\exp \left(\operatorname{Höl}_{\alpha}\left(g^{\prime}\right) /\left(\lambda(g)^{\alpha}-1\right)\right)$ which is a consequence of

$$
\left|v_{i_{k} \cdots i_{n}}^{g}-u_{i_{k} \cdots i_{n}}^{g}\right| \leq \lambda(g)^{n-k+1} .
$$

We conclude the proof. Given $x, y \in S^{1}$ there exists $z \in[x, y]$ such that $[x, z]$ (respectively $[z, y]$ ) can be covered by an interval of the form $\left[z_{i}^{g}, z_{i+1}^{g}\right]$ of order $n$ and simultaneously contains at least an interval of the form $\left[z_{i j}^{g}, z_{i j+1}^{g}\right]$ of order $n+1$ ( $n$ need not be the same for $x$ and $y$ ). Then

$$
\begin{aligned}
d(\theta(x), \theta(z)) & \leq\left|\bar{z}_{i}^{f}-\bar{z}_{i+1}^{f}\right| \leq\left|\bar{z}_{i}^{g}-\bar{z}_{i+1}^{g}\right|^{\gamma} \\
& \leq\left(\frac{1}{2} C(g)\right)^{\gamma}\left|\bar{z}_{i j}^{g}-\bar{z}_{i j+1}^{g}\right|^{\gamma} \leq \frac{1}{2} C(g) d(x, y)^{\gamma}
\end{aligned}
$$

and an equivalent estimate holds for $d(\theta(z), \theta(y))$. To obtain the other inequality, we use instead

$$
\left|\bar{z}_{i j}^{g}-\bar{z}_{i j+1}^{g}\right| \leq\left|\bar{z}_{i j}^{f}-\bar{z}_{i j+1}^{f}\right|^{\gamma} \leq d(\theta(x), \theta(z))^{\gamma} .
$$

In the next lemma, we show that any $\mathcal{C}^{1+\beta}$-map of the circle can be approximated by smooth maps, in the $\mathcal{C}^{1+\alpha}$-topology, for any $\alpha<\beta$ (and not in the $\mathcal{C}^{1+\beta}$-topology). For the purposes of this paper we need a more precise statement.

LEMMA 27. For any $0<\alpha<1$, the space $\mathcal{F}_{\alpha} \cap \mathcal{C}^{\infty}$ of smooth maps of $S^{1}$ is dense in $\mathcal{F}_{\alpha+}$ in the $\mathcal{C}^{1+\alpha}$-topology.

Proof. Let $f \in \mathcal{F}_{\beta}$ for some $\beta>\alpha, \bar{f}$ be the lift to $\mathbb{R}$ of $f,\left(\rho_{n}\right)$ be a smooth approximation of unity (i.e. $\int \rho_{n}(t) d t=1$, the support of $\rho_{n}$ is included in ] $-1 / n, 1 / n[$ ) and $\bar{f}_{n}=\bar{f} * \rho_{n}-\bar{f} * \rho_{n}(0)$, the convolution of $f$ with $\rho_{n}$. Then $\bar{f}_{n}$ is a smooth map, $\bar{f}_{n}(x+1)=\bar{f}_{n}(x)+D, \bar{f}_{n}(0)=0$ and $\lambda\left(f_{n}\right) \geq \lambda(f)$. The sequence of maps $\left(\bar{f}_{n}\right)$ converge to $\bar{f}$ in the $\mathcal{C}^{1}$-topology. In particular, for large $n, f_{n}$ is a smooth covering of degree $D$ of the circle, preserves orientation and satisfies the same constraints for the first derivative. Moreover, the $\beta$-Hölder semi-norm of $f_{n}^{\prime}$ is uniformly bounded:

$$
\begin{aligned}
\left|f_{n}^{\prime}(x)-f_{n}^{\prime}(y)\right| & \leq \int\left|f^{\prime}(x-t)-f^{\prime}(y-t)\right| \rho(t) d t \\
& \leq \operatorname{Höl}_{\beta}\left(f^{\prime}\right)|x-y|^{\beta} .
\end{aligned}
$$

By using Lemma $17,\left(f_{n}^{\prime}\right)$ converges to $f^{\prime}$ in the $\mathcal{C}^{\alpha}$-topology for any $\alpha<\beta$, and $\left(f_{n}\right)$ converges to $f$ in the $\mathcal{C}^{1+\alpha}$ topology.

We now give a short summary of the theory of the thermodynamic formalism for any $T \in \mathcal{F}_{\alpha}$, which are particular expanding Markov maps. An interested reader may find a comprehensive exposition of this theory in [20]. Given a potential $A: S^{1} \rightarrow \mathbb{R}$, we define 
the Ruelle-Perron-Frobenius operator $\mathcal{L}_{A}$, acting on the set of continuous functions, by the following formula:

$$
\left(\mathcal{L}_{A} \cdot h\right)(x)=\sum_{T(y)=x} h(y) e^{A(y)}, \quad \forall x \in S^{1}
$$

where $h: S^{1} \rightarrow \mathbb{R}$ is a continuous function and the summation is taken over the $D$ preimages of $x$. If $A$ is $\alpha$-Hölder, $\mathcal{L}_{A}$ also acts on $\alpha$-Hölder functions and, by duality, $\mathcal{L}_{A}$ acts on the set of probability measures by

$$
\int h d\left(\mathcal{L}_{A}^{*} \cdot \mu\right)=\int \mathcal{L}_{A} \cdot h d \mu
$$

for any continuous test functions $h$. For such Markov expanding and mixing map $T$ and for such $\alpha$-Hölder potentials $A$, the spectrum $\sigma\left(\mathcal{L}_{A}\right)$ of $\mathcal{L}_{A}$, acting on $\mathcal{C}^{\alpha}$-potentials, admits an isolated simple eigenvalue $e^{P_{T}(A)}$ which dominates the whole spectrum. $P_{T}(A)$ is called the pressure of $A$ and the rest of the spectrum has a strictly smaller modulus:

$$
\sup _{z \neq \exp P_{T}(A)}\left\{|z| \mid z \in \sigma\left(\mathcal{L}_{A}\right)\right\}<\exp P_{T}(A) .
$$

Moreover, there exists a unique probability measure $\mu_{A}$, called the Gibbs measure, and a unique positive $\alpha$-Hölder function $h_{A}$ such that

$$
\mathcal{L}_{A}^{*} \cdot \mu_{A}=e^{P_{T}(A)} \mu_{A}, \quad \mathcal{L}_{A} \cdot h_{A}=e^{P_{T}(A)} h_{A} \quad \text { and } \quad \int h_{A} d \mu_{A}=1 .
$$

We also recall that a map $T$ possesses a Jacobian $\left.J: S^{1} \rightarrow\right] 0,+\infty[$ with respect to $\mu$ if

$$
\mu(T(U))=\int_{U} J d \mu
$$

for all the Borel set $U$ where $T: U \rightarrow S^{1}$ is one-to-one. Then $e^{P_{T}(A)-A}$ is the Jacobian of $T$ with respect to $\mu_{A}$. The measure $\mu_{A}^{\text {equi }}=h_{A} \mu_{A}$ is called the equilibrium measure associated to $A$, it is $T$-invariant and

$$
\frac{h_{A}}{h_{A} \circ T} \exp \left(P_{T}(A)-A\right)
$$

is the Jacobian of $T$ with respect to $\mu_{A}^{\text {equi }}$.

Although the following proposition is certainly well known, the thermodynamic formalism gives simple criteria for $\ln f^{\prime}$ to be cohomologous to a constant and the existence of a sub-action simplifies the proof.

Proposition 28. Let $f \in \mathcal{F}_{\alpha}$. The following properties are equivalent:

(i) $\ln f^{\prime}$ is measurably cohomologous to a constant (the constant is actually equal to $\ln D$ );

(ii) for any periodic point $x$ of period $p,\left(f^{p}\right)^{\prime}(x)=D^{p}$;

(iii) $f$ is $\mathcal{C}^{1}$-conjugate to $T(z)=z^{D}$ (if $f(1)=1$ the conjugacy is actually unique and $\mathcal{C}^{1+\alpha}$; moreover, $\ln f^{\prime}$ is equal to an Hölder coboundary);

(iv) if $\mu$ denotes the unique absolutely continuous invariant probability, then $h_{\mu}(f)=$ $h_{\text {top }}(f)$. 
Proof. We first notice that Pesin's formula gives $h_{\mu}(f)=\int \ln f^{\prime} d \mu$, besides $h_{\text {top }}(f)=$ $\ln D$.

(i) $\Rightarrow$ (iv) uses the fact that, if a coboundary $V \circ f-V$ is integrable, then $\int(V \circ f-$ V) $d \mu=0$.

(iv) $\Rightarrow$ (iii) uses Jensen's inequality: let $h$ be the unique eigenfunction, $\mathcal{L}_{A}(h)=h$, for the Ruelle-Perron-Frobenius operator associated to $A=-\ln f^{\prime}$ normalized by $\int h d \mu=1$. Then $\mu=h$ Leb and

$$
\ln D=\int \ln f^{\prime} d \mu=\int \ln \left(f^{\prime} h \circ f / h\right) d \mu \leq \ln \left[\int f^{\prime}(h \circ f / h) d \mu\right]=\ln D
$$

(we have used $\int \phi \mathcal{L}_{A}(\psi) d$ Leb $=\int \phi \circ f \psi d$ Leb and $\mathcal{L}_{A}\left(f^{\prime}\right)=D$ ). By strict convexity of ln, we get $h \circ f f^{\prime}=D h$. If $\bar{h}$ is a lift of $h$ and $\bar{\theta}(x)=\int_{0}^{x} \bar{h}(t) d t$ then $\theta \circ f=T \circ \theta$.

(iii) $\Rightarrow$ (ii) is trivial.

(ii) $\Rightarrow$ (i) uses the fact that periodic measures are dense (for the weak topology) in the space of ergodic invariant probability measures. Then

$$
\min _{\mu} \int \ln f^{\prime} d \mu=\max _{\mu} \int \ln f^{\prime} d \mu=\ln D
$$

where we minimize (maximize) over the set of invariant probability measures. Thanks to Proposition 11, we can find Hölder functions $V, W$ such that

$$
\ln D+W \circ f-W \geq \ln f^{\prime} \geq \ln D+V \circ f-V .
$$

$W-V$ is increasing along the orbits and is therefore constant everywhere. We have therefore shown $\ln f^{\prime}=\ln D+V \circ f-V$.

Using the thermodynamic formalism, we are now able to give another construction of maximizing measures and sub-actions. We recall that an equilibrium measure gives positive mass to any non-empty open set and is therefore never a maximizing measure for a potential which is not cohomologous to a constant. Part (i) of the next proposition appeared in a slightly different form in [16]. Part (iii) was suggested to the last author by M. Pollicott from a communication of S. V. Savchenko.

Proposition 29. Let $f$ be expanding and A be a $\alpha$-Hölder potential. Then

(i) any weak limit $\mu$ of $\left(\mu_{t A}^{\text {equi }}\right)$ when $t$ goes to $+\infty$ is a maximizing measure for $A$;

(ii) the metric entropy of $h\left(\mu_{t A}^{\text {equi }}\right)$ converges to $h_{\text {top }}(f \mid \Omega(A, f))$ when $t$ goes to $+\infty$; in particular, any weak limit $\mu$ is of maximal entropy for $f$ restricted to $\Omega(A, f)$;

(iii) rewrite $h_{t A}=\exp \left(t V_{t}\right)$, the unique eigenfunction of $\mathcal{L}_{t A}$ for the eigenvalue $\exp P_{T}(t A)$ normalized by $\int h_{t A} d \mu_{t A}^{\text {equi }}=1$. Then the sequence $\left(V_{t}\right)_{t>0}$ stays in a compact set in the uniform topology and any accumulation function $V$ gives a sub-action satisfying

$$
V(x)=\max _{T(y)=x}(V(y)+A(y)-m) .
$$


Proof. To simplify, we denote $\mu_{t}=\mu_{t A}^{\text {equi }}$. For any $f$-invariant measure $\mu$

$$
t \int A d \mu \leq h(\mu)+t \int A d \mu \leq h\left(\mu_{t}\right)+t \int A d \mu_{t} \leq h_{\text {top }}+t \int A d \mu_{t} .
$$

We obtain, therefore, for any maximizing measure $\mu \in \mathcal{M}(A, f)$

$$
\left|\int A d \mu-\int A d \mu_{t}\right| \leq \frac{1}{t} h_{\text {top }}(T) \text { and } h(\mu) \leq h\left(\mu_{t}\right)
$$

which shows the first assertion. On the one hand,

$$
h_{\text {top }}(f \mid \Omega(A, f)) \leq \inf _{t>0} h\left(\mu_{t}\right) .
$$

On the other hand, $\mu \rightarrow h(\mu)$ is upper semi-continuous and

$$
\limsup _{t \rightarrow+\infty} h\left(\mu_{t}\right) \leq h_{\text {top }}(f \mid \Omega(A, f)) .
$$

To simplify again, we denote $\mathcal{L}_{t}=\mathcal{L}_{t A-P_{T}(t A)}$. For every $n$, define $V_{t}^{n}$ by $\mathcal{L}_{t} 1=\exp \left(t V_{t}^{n}\right)$ for $t>0$ and $V_{0}^{n}=0$ by convention. Since $\mathcal{L}_{t}$ leaves invariant the following compact set of functions (for the uniform topology)

$$
\mathcal{S}_{t}=\left\{h \in \mathcal{C}^{0}\left(S^{1}\right) \mid h \geq 0, \int h d \mu_{t}=1, h(x) \leq h(y) \exp \left(C_{t} d(x, y)^{\alpha}\right), \forall x, y\right\}
$$

where

$$
C_{t}=\operatorname{Höl}_{\alpha}\left(t A-P_{T}(t A)\right) /\left(\lambda^{\alpha}-1\right)=t \operatorname{Höl}_{\alpha}(A) /\left(\lambda^{\alpha}-1\right),
$$

we obtain for all $t \geq 0$ and for all $n \geq 0$

$$
\operatorname{Höl}_{\alpha}\left(V_{t}^{n}\right) \leq \operatorname{Höl}_{\alpha}(A) /\left(\lambda^{\alpha}-1\right) .
$$

Since $\left(\mathcal{L}_{t}^{n} 1\right)_{n \geq 0}$ converges uniformly to $h_{t}=h_{t A-P_{T}(A)}>0, \quad\left(V_{t}^{n}\right)_{n \geq 0}$ converges uniformly to some $V_{t}$ with the same Hölder coefficient $\operatorname{Höl}_{\alpha}(A) /\left(\lambda^{\alpha}-1\right)$. The eigenfunction $h_{t}=\exp \left(t V_{t}\right)$ is a solution of $\mathcal{L}_{t} h_{t}=h_{t}$ or $\mathcal{L}_{t}\left(h_{t} / h_{t} \circ T\right)=1$. We first obtain (for $t>0$ )

$$
t R_{t} \stackrel{\text { def }}{=} t\left(V_{t} \circ T-V_{t}\right)-t A+P_{T}(t A)>0 .
$$

The definition of pressure implies $\operatorname{tm}(A) \leq P_{T}(t A) \leq h_{\text {top }}(T)+\operatorname{tm}(A)$. By letting $t$ go to infinity, any accumulation limit $V$ of $\left(V_{t}\right)$ is a sub-action. Since $t R_{t}$ satisfies more precisely the functional equation,

$$
\exp \left(-t R_{t}\left(x_{1}\right)\right)+\cdots+\exp \left(-t R_{t}\left(x_{D}\right)\right)=1
$$

for any $x_{1}, \ldots, x_{D}$ with the same image. When $t$ goes to infinity, a sub-sequence of $\left(R_{t}\right)_{t>0}$ converges to $R=V \circ T-V-A+m(A)$ with

$$
\min \left(R\left(x_{1}\right), \ldots, R\left(x_{D}\right)\right)=0 .
$$

In the following lemma we define a kind of 'local' chart $\left(\mathcal{U}_{T}, \Phi_{T}\right)$ about any $T \in \mathcal{F}_{\alpha+}$. The difficulty comes from the loss of differentiability in the conjugating map $\theta$ which is not Lipschitz but $\gamma$-Hölder with $\gamma$ as close to one as we want provided $\mathcal{U}_{T}$ is small enough. 
Lemma 30. Let $0<\alpha<\beta, T \in \mathcal{F}_{\beta}$ and $A=-\ln T^{\prime}$.

(i) There exists a $\mathcal{C}^{1+\beta}$-neighborhood $\tilde{\mathcal{U}}_{T}$ of $T$ such that $\mathcal{P}(f)=-\ln f^{\prime} \circ \theta_{f, T}$ is a $\mathcal{C}^{\alpha}$-function depending continuously on $f \in \tilde{\mathcal{U}}_{T}$.

(ii) Conversely, there exist a $\mathcal{C}^{\beta}$-neighborhood $\mathcal{U}_{T}$ of $A$ and a continuous map $\Phi_{T}$ : $\mathcal{U}_{T} \rightarrow \mathcal{F}_{\alpha+}$ such that for every $F \in \mathcal{U}_{T}, f=\Phi_{T}(F)$ is the solution of the equation,

$$
F-P_{T}(F)=-\ln f^{\prime} \circ \theta_{f, T} .
$$

Moreover, $\mathcal{U}_{T}$ can be any neighborhood of the form

$$
\mathcal{U}_{T}=\left\{F \in \mathcal{C}^{\beta} \mid \operatorname{Höl}_{\beta}(F)<C \text { and }\|F-A\|_{0}<\epsilon\right\}
$$

where $C$ is any positive constant and $\epsilon=\epsilon(C)$ is sufficiently small.

Proof. Part $(i)$. Since $\theta_{f}$ is $\gamma(f, T)$-Hölder and can be as close to one as desired, we choose $\gamma^{*}<1$ such that $\beta \gamma^{*}>\alpha$. We also choose $C^{*}>\operatorname{Höl}_{\beta}\left(T^{\prime}\right), \lambda^{*}<\lambda(T), \Lambda^{*}>\Lambda(T)$ and

$$
\tilde{\mathcal{U}}_{T}=\left\{f \in \mathcal{F}_{\beta} \mid \gamma(f, T)>\gamma^{*}, \operatorname{Höl}_{\beta}\left(f^{\prime}\right)<C^{*}, \lambda(f)>\lambda^{*} \text { and } \Lambda(f)<\Lambda^{*}\right\} .
$$

For any $f \in \tilde{\mathcal{U}}_{T}$, the $\beta \gamma^{*}$-Hölder norm of $F=\mathcal{P}(f)$ is bounded by

$$
C^{*}\left[2 D \exp \left(C^{*} /\left(\lambda^{* \beta}-1\right)\right]^{\beta}\right. \text {. }
$$

For any $f, g$ in $\tilde{\mathcal{U}}_{T}, F=\mathcal{P}(f), G=\mathcal{P}(g)$,

$$
|F(x)-G(x)| \leq\left|f^{\prime} \circ \theta_{f}(x)-g^{\prime} \circ \theta_{g}(x)\right|
$$

(we have used the fact that $\left|f^{\prime}\right|>\lambda>1$ ), and by using Lemma 26 we obtain

$$
\|A-B\|_{0} \leq\left\|f^{\prime}-g^{\prime}\right\|_{0}+\frac{\Lambda^{*}}{\lambda^{*}-1}\|\bar{f}-\bar{g}\|_{0} .
$$

Continuity of $\mathcal{P}$ now follows from Lemma 17.

Part (ii). For any $\mathcal{C}^{\beta}$-function $F, \exp \left(P_{T}(F)-F\right)$ is the Jacobian of $T$ with respect to the Gibbs measure $\mu_{F}$. If $\theta_{F}: S^{1} \rightarrow S^{1}$ is a homeomorphism, $\exp \left(P_{T}(F)-F\right) \circ \theta_{F}^{-1}$ is the Jacobian of $f=\theta_{F} \circ T \circ \theta_{F}^{-1}$ with respect to the push measure $\left(\theta_{F}\right)_{*} \mu_{F}$. We are therefore looking for a $\theta_{F}$ which satisfies $\left(\theta_{F}\right)_{*} \mu_{F}=$ Leb and $\theta_{F}(1)=1$. The only possibility for $\theta_{F}$ is given by the formula

$$
\operatorname{Leb}\left(\bar{\theta}_{F}[0, x]\right)=\bar{\theta}_{F}(x)=\bar{\mu}_{F}([0, x]), \quad \forall x \in[0,1[
$$

where $\bar{\theta}_{F}$ is the lift of $\theta_{F}$ and $\bar{\mu}_{F}$ is the corresponding measure on [0,1[. Since $\mu_{F}$ gives positive measure to non-empty open sets and has no mass, $\theta_{F}: S^{1} \rightarrow S^{1}$ is an homeomorphism. Let $f=\theta_{F} \circ T \circ \theta_{F}^{-1}$, then $f$ is a covering of degree $D$ and preserves the orientation. Moreover, $J=\exp \left(P_{T}(F)-F \circ \theta_{F}^{-1}\right)$ is the Jacobian of $f$ with respect to the Lebesgue measure and

$$
\bar{f}(y)-\bar{f}(x)=\int_{x}^{y} \bar{J}(t) d t
$$

for any $0<x<y<1$. This shows in particular that $f$ is $\mathcal{C}^{1}$ and

$$
-\ln f^{\prime} \circ \theta_{F}=F-P_{T}(F) .
$$


By Lemma 26 (with $\beta$ instead of $\alpha$ ), $\theta_{f}=\theta_{F}, \theta_{F}^{-1}$ is $\gamma(T, f)$-Hölder and its $\gamma$-Hölder constant is bounded by $C(T)$. Then $f^{\prime}$ is $\beta \gamma$-Hölder and

$$
\mathrm{Höl}_{\beta \gamma}\left(f^{\prime}\right) \leq\left\|\exp \left(P_{T}(F)-F\right)\right\|_{0} \operatorname{Höl}_{\beta}(F) C(T)^{\beta} \text {. }
$$

Let $\gamma^{*}<1$, close to 1 , such that $\beta \gamma^{*}>\alpha$. Let $C^{*}>\operatorname{Höl}_{\beta}(A)$. Let $\lambda^{*}<\Lambda^{*}$ such that $\lambda^{*}<\lambda(T)$ and $\Lambda^{*}>\Lambda(T)=\max _{x \in S^{1}} T^{\prime}(x)$. Define for small $\epsilon$,

$$
\mathcal{U}_{T}=\left\{F \in \mathcal{C}^{\beta} \mid \operatorname{Höl}_{\beta}(F)<C^{*} \text { and }\|F-A\|_{0}<\epsilon\right\} .
$$

For small $\epsilon$ we get

$$
\max _{F \in \mathcal{U}_{T}} \max _{x \in S^{1}} \exp \left(P_{T}(F)-F(x)\right) \leq \Lambda^{*}, \quad \min _{F \in \mathcal{U}_{T}} \min _{x \in S^{1}} \exp \left(P_{T}(F)-F(x)\right) \geq \lambda^{*} .
$$

We also get $\gamma(T, f) \geq \gamma^{*}$ and a uniform upper bound for the $\beta \gamma^{*}$-Hölder constant of $f^{\prime}$. If we can show that $f^{\prime}$ depends continuously with respect to $F$ in the uniform topology, using Lemma 17, we prove the continuity of $\Phi_{T}(F)$ with respect to $F \in \mathcal{U}_{T}$ as a $\mathcal{C}^{1+\alpha}$ maps.

We first notice the following estimate:

$$
\|\bar{f}-\bar{g}\|_{0} \leq\left\|f^{\prime}-g^{\prime}\right\|_{0}
$$

where $f^{\prime}=\exp \left(P_{T}(F)-F\right) \circ \theta_{F}^{-1}, g^{\prime}=\exp \left(P_{T}(G)-G\right) \circ \theta_{G}^{-1}$. Then we have

$$
\begin{gathered}
\left\|f^{\prime}-g^{\prime}\right\|_{0} \leq \Lambda^{*}\left[2\|F-G\|_{0}+C^{*}\left\|\left(\bar{\theta}_{F}\right)^{-1}-\left(\bar{\theta}_{G}\right)^{-1}\right\|_{0}^{\beta}\right] \\
\left\|\left(\bar{\theta}_{F}\right)^{-1}-\left(\bar{\theta}_{G}\right)^{-1}\right\|_{0} \leq C(T)\left\|\bar{\theta}_{F}-\bar{\theta}_{G}\right\|_{0}^{\ln \lambda^{*} / \ln \Lambda^{*}} .
\end{gathered}
$$

To conclude, it is now enough to prove that $\bar{\theta}_{F}$ depends continuously with respect to $F \in \mathcal{U}_{T}$ in the uniform topology.

Let $\left(F_{n}\right)$ be a sequence of $\beta$-Hölder functions converging to $F$ in the $\mathcal{C}^{\beta}$-topology and $\left(f_{n}\right)$ the corresponding maps. We show that $\left(\bar{\theta}_{F_{n}}\right)$ converges to $\bar{\theta}_{F}$ uniformly. Restricted to $[0,1], \bar{\theta}_{F_{n}}$ corresponds to the distribution of the measure $\bar{\mu}_{F_{n}}$. Since $\bar{\theta}_{F}, \bar{\theta}_{F_{n}}$ are increasing and continuous, by using Helly's theorem, it is enough to show that $\left(\bar{\theta}_{F_{n}}\right)$ converges pointwise to $\bar{\theta}_{F}$ or that $\left(\bar{\mu}_{F_{n}}\right)$ converges weakly to $\bar{\mu}_{F}$ (we use here the fact that $\bar{\mu}_{A}$ has no atom).

Let $v$ be a weak limit of some sub-sequence $\left(\mu_{F_{n^{\prime}}}\right)$. Since $\mathcal{L}_{F_{n}}$ converges to $\mathcal{L}_{F}$ in the uniform topology, for any test function $h: S^{1} \rightarrow \mathbb{R}$,

$$
\begin{gathered}
\lim _{n^{\prime} \rightarrow \infty} \int \mathcal{L}_{F_{n^{\prime}}} \cdot h d \mu_{F_{n^{\prime}}}=\int \mathcal{L}_{F} \cdot h d v \\
\int \mathcal{L}_{F_{n^{\prime}}} \cdot h d \mu_{F_{n^{\prime}}}=e^{P\left(F_{n^{\prime}}\right)} \int h d \mu_{F_{n^{\prime}}} \\
\lim _{n^{\prime} \rightarrow \infty} e^{P\left(B_{n^{\prime}}\right)} \int h d \mu_{B_{n^{\prime}}}=e^{P(F)} \int h d \nu .
\end{gathered}
$$

We thus obtain that $v$ is solution of $\mathcal{L}_{F} \cdot v=e^{P(F)} v$, and by uniqueness of Gibbs measures, $v=\mu_{F}$. Since $\gamma(T, f)$ depends continuously with respect to $f$ in the $\mathcal{C}^{1}$-topology, $\gamma(T, f)$ can be made larger than $\gamma^{*}$ for every $F \in \mathcal{U}_{T}$ as soon as $\epsilon$ is chosen small enough. 
Remark 31. In the previous lemma, $\left(\mathcal{U}_{T}, \Phi_{T}\right)$ is not a one-to-one parametrization. Although we do not need it, we could have been more precise. The set $\mathcal{P}=\{A \in$ $\mathcal{C}^{\alpha} \mid A<0$ and $\left.P_{T}(A)=0\right\}$ is actually an embedded $\mathcal{C}^{1}$-manifold: for each $A \in \mathcal{P}$, $\mathcal{P}$ is homeomorphic to an open set in a Banach space $\mathcal{T}_{A}=\left\{F \in \mathcal{C}^{\alpha} \mid F<0\right.$ and $\left.\int(F-A) d \mu_{A}^{\text {equi }}=0\right\}$. Indeed if $F \in \mathcal{P}$,

$$
\frac{\int A d \mu_{A}^{\text {equi }}}{\int F d \mu_{A}^{\text {equi }}} F \in \mathcal{T}_{A} \quad \text { and } \quad \frac{\int A d \mu_{A}^{\text {equi }}}{\int F d \mu_{A}^{\text {equi }}} \geq 1
$$

where $\mu_{A}^{\text {equi }}$ is the equilibrium measure associated to $A$. Conversely, if $F \in \mathcal{T}_{A}$, the pressure $P$ is $\mathcal{C}^{1}$-differentiable on the space of $\mathcal{C}^{\alpha}$-potentials (see [17]) and

$$
P_{T}^{\prime}(A) . H=\int H d \mu_{A}^{\text {equi }}
$$

The map $p(t, F)=P_{T}(t F)$ is therefore $\mathcal{C}^{1}$ with respect to $t$ and $F$,

$$
\frac{\partial p}{\partial t}=\int F d \mu_{F}^{\text {equi }}<0, \quad p(0, F)=\ln D \quad \text { and } \quad p(1, F) \leq 0 .
$$

The function $p(t, F)$ is decreasing with respect to $t$, and there exists a unique $0<\delta(F) \leq 1$ such that $p(\delta(F), F)=0$. By the implicit function theorem, the function $F \mapsto \delta(F)$ is $\mathcal{C}^{1}$.

Proof of Theorem 2. Let $\mathcal{G}$ be the set of maps $f$ in $\mathcal{F}_{\alpha}$ admitting a unique Lyapunov minimizing measure supported on a periodic orbit and satisfying the property of continuously varying support. We first show that $\mathcal{G}$ is open in $\mathcal{F}_{\alpha}$ and that $\mathcal{G}_{+}=\mathcal{G} \cap \mathcal{F}_{\alpha+}$ is dense in $\mathcal{F}_{\alpha+}(\lambda, \Lambda)$.

To show that $\mathcal{G}$ is open, we proceed as in the proof of Theorem 8(ii). Let $f_{0} \in \mathcal{G}$, let

$$
\mu_{0}=\frac{1}{N} \sum_{k=0}^{N-1} \delta_{f_{0}^{k}\left(p_{0}\right)}
$$

be the unique minimizing periodic measure, $\mathcal{N}$ a neighborhood of the orbit $\operatorname{orb}\left(p_{0}\right)=$ $\left\{p_{0}, f_{0}\left(p_{0}\right), \ldots, f_{0}^{N}\left(p_{0}\right)=p_{0}\right\}$ and $\mathcal{U}$ a neighborhood of $f_{0}$ in the $\mathcal{C}^{1+\alpha}$-topology such that, for any $f \in \mathcal{U}, f$ has a unique periodic orbit $p$ of period $N$ in $\mathcal{N}$ which is also the only $f$-invariant compact set in $\mathcal{N}$. We note that

$$
\mu_{f}=\frac{1}{N} \sum_{k=0}^{N-1} \delta_{f^{k}(p)}
$$

be the corresponding periodic measure. Thanks to the property of continuously varying support, $\mathcal{U}$ can be chosen small enough so that, for any $f \in \mathcal{U}$, any Lyapunov minimizing measure $\mu$ for $f$ has a support included in $\mathcal{N}$ and is therefore equal to $\mu_{f}$. To prove that any $f \in \mathcal{U}$ also satisfies the property of continuously varying support, we choose $\left(f_{n}\right)$ converging to $f$ in the uniform topology and notice that any accumulation set $K$ of the sequence $\left(\operatorname{supp}\left(\mu_{f_{n}}\right)\right)$ is equal to the unique periodic orbit of $f$ in $\mathcal{N}$.

We now show that $\mathcal{G}_{+}$is dense in $\mathcal{F}_{\alpha+}$. Let $T \in \mathcal{F}_{\alpha+}$, then $T \in \mathcal{F}_{\beta}$ for some $\beta>\alpha$ and $A=-\ln T^{\prime}$ is $\mathcal{C}^{\beta}$. According to Theorem 8 (and more precisely its proof) there 
exists a sequence $\left(F_{n}\right)$ of $\beta$-Hölder functions having a $\beta$-Hölder norm uniformly bounded converging to $A$ in the $\mathcal{C}^{0}$-topology and such that $F_{n} \in \mathcal{G}_{+}$for all $n$. Thanks to Lemma 30, $\left(\Phi_{T}\left(F_{n}\right)\right)$ converges to $T$ as $\mathcal{C}^{1+\alpha}$-maps and the set of Lyapunov minimizing measures of $\Phi_{T}\left(F_{n}\right)$ coincides with the set of maximizing measures of $F_{n}$.

The proof of the second part of Theorem 2 follows from the equivalence between (i) and (ii) in Proposition 20.

Acknowledgements. We would like to thank the referee for a careful reading and valuable comments on this article.

GC was partially supported by Conacyt-Mexico grant 28489-E. AOL was partially supported by PRONEX-CNPq - Sistemas Dinâmicas and PhT was partially supported by CNRS URA 1169.

\section{REFERENCES}

[1] G. Atkinson. Recurrence of cocycles and random walks. J. London Math. Soc. 13(2) (1976), 486-488.

[2] Th. Bousch. Le poisson n'a pas d'arêtes. Ann. Inst. Henri Poincaré 36 (2000), 489-508.

[3] R. Bowen. Entropy for maps of the interval. J. Topology 16 (1977), 465-467.

[4] M. Craizer. MSc Thesis, IMPA.

[5] M. J. Dias-Carneiro. On minimizing measures of the action of autonomous Lagrangians. Nonlinearity 8 (1995), 1077-1085.

[6] G. Contreras, J. Delgado and R. Iturriaga. Lagrangian flows: the dynamics of globally minimizing orbits-II. Bol. Soc. Bras. Mat. 28(2), (1997), 155-196.

[7] G. Contreras, R. Iturriaga, G. P. Paternain and M. Paternain. Lagrangian graphs, minimizing measures and Mañé's critical values. Geom. Funct. Anal. 8(2) (1998), 417-444.

[8] A. Fathi. Théorème KAM faible et théorie de Mather sur les systèmes lagrangiens. C. R. Acad. Sci. Paris, I. 324 (1997), 1043-1046.

[9] G. Forni and J. Mather. Action Minimizing Orbits in Hamiltonian Systems (Lecture Notes in Mathematics, 1589). Springer, 1994, pp. 92-186.

[10] C. Grillenberger. Constructions of strictly ergodic systems I. Given entropy. Z. Wahrsch. Verw. Gebiete 25 (1973), 323-334.

[11] C. Grillenberger. Constructions of strictly ergodic systems II. K-systems. Z. Wahrsch. Verw. Gebiete 25 (1973), 335-342.

[12] B. R. Hunt. Maximal local Lyapunov dimension bounds the box dimension of chaotic attractors. Nonlinearity 9 (1996), 845-852.

[13] Y. Guocheng and B. R. Hunt. Optimal orbits of hyperbolic systems. Preprint.

[14] O. M. Jenkinson. Frequency-locking on the boundary of the barycenter set. Exp. Math. 9 (2000), 309-317.

[15] O. M. Jenkinson. On the barycentre of invariant measures for circle maps. Preprint.

[16] A. Lopes. Dimension spectra and a mathematical model for phase transitions. Adv. Appl. Math. 11(4) (1990), 475-502.

[17] R. Mañe. The Hausdorff dimension of horseshoes of diffeomorphisms of surfaces. Bol. Soc. Bras. Mat. 20(2) (1990), 1-24.

[18] R. Mañe. Generic properties and problems of minimizing measures of Lagrangian systems. Nonlinearity 9 (1996), 273-310.

[19] R. Mañé. Lagrangian flows: the dynamics of globally minimizing orbits. Int. Congress on Dyn. Sys. in Montevideo (a tribute to Ricardo Mañé) (Pitman Research Notes in Mathematics, 362). Eds F. Ledrappier, J. L. Lewowicz and S. Newhouse. Longman, Harlow, 1996, pp. 120-131.

Reprinted in Bol. Soc. Bras. Mat. 28(2) (1995), 141-153.

[20] W. Parry and M. Pollicott. Zeta function and the periodic orbit structure of hyperbolic dynamics. Asterisque 187-188, 1990. 
[21] R. Rockafellar. Convex Analysis. Princeton University Press, 1970.

[22] $\mathrm{Ph}$. Thieullen. Entropy and the Hausdorff dimension for infinite-dimensional dynamical systems. J. Dynam. Diff. Eqn. 4(1) (1992), 127-159

[23] A. Loyes and $\mathrm{Ph}$. Thieullen. Sub-actions for Anosov diffeomorphisms. Preprint. 